\title{
ESSENTIAL MODIFICATIONS ON BIOGEOGRAPHY-BASED OPTIMIZATION ALGORITHM
}

\author{
Ali R. Alroomi ${ }^{1}$, Fadhel A. Albasri ${ }^{2}$ and Jawad H. Talaq ${ }^{3}$ \\ ${ }^{1}$ Electrical Engineer, University of Bahrain, Sakhir, Bahrain \\ wadyan@gmail.com \\ ${ }^{2}$ Department of Electrical Engineering, University of Bahrain, Sakhir, Bahrain \\ falbasri@uob.edu.bh \\ ${ }^{3}$ Department of Electrical Engineering, University of Bahrain, Sakhir, Bahrain \\ jhstallaqdeng.uob.bh
}

\begin{abstract}
Biogeography-based optimization (BBO) is a new population-based evolutionary algorithm and is based on an old theory of island biogeography that explains the geographical distribution of biological organisms. BBO was introduced in 2008 and then a lot of modifications were employed to enhance its performance. This paper proposes two modifications; firstly, modifying the probabilistic selection process of the migration and mutation stages to give a fairly randomized selection for all the features of the islands. Secondly, the clear duplication process after the mutation stage is sized to avoid any corruption on the suitability index variables. The obtained results through wide variety range of test functions with different dimensions and complexities proved that the BBO performance can be enhanced effectively without using any complicated form of the immigration and emigration rates. This essential modification has to be considered as an initial step for any other modification.
\end{abstract}

\section{KEYWORDS}

Biogeography-Based Optimization, BBO, Evolutionary Algorithm, Migration, Partial Migration

\section{INTRODUCTION}

The science of biology becomes one of the main resources of inspiration to develop the modern optimization techniques, such as ant colony optimization (ACO), bee colony optimization (BCO), wasp swarm optimization (WSO), bacterial foraging optimization (BFO), genetic algorithm (GA), evolutionary strategy (ES), differential evolution (DE), particle swarm optimization (PSO), etc. Biogeography-based optimization (BBO) is a new population-based evolutionary algorithm (EA) that was introduced by Dan Simon in 2008 [1].

The algorithm of BBO is based on an old theory of island biogeography that explains the geographical distribution of biological organisms. This theory was established by $\mathrm{H}$. MacArthur and Edward O. Wilson during their exploration study in the period between 1960-1967 [2,3]. 
Island, in biogeography, is any area of suitable habitat (local environment occupied by an organism [5]) surrounded by an expense of unsuitable habitat and is endowed with exceptionally rich reservoirs of endemic, exclusive, strange and relict species [6].

Each island has its own features as simple biotas, varying combinations of biotic and abiotic factors, and variability in isolation, shape, and size [7,9].

In $\mathrm{BBO}$, the islands (or habitats) are the dependent variables, and the features availability on those islands represents the independent variables.

The original $\mathrm{BBO}$ has a weakness in its migration and mutation stages that affects its performance. The objective of this paper is to solve that weakness by focusing on the root problems instead of using other complicated approaches, such as the complicated migration models represented in $[13,16]$.

This paper is organized as follows: Section 2 gives a brief overview about the theory of island biogeography and Section 3 explains the algorithm of BBO. Section 4 shows how BBO performance can be enhanced more by solving the root problems of the migration and mutation stages, and then followed by a performance comparison between the original and the modified BBO in Section 5. The conclusions and suggestions are presented inSection 6.

\section{THE THEORY OF ISLAND BIOGEOGRAPHY}

The equilibrium theory of island biogeography proposes that the number of inhabited species on an island is based on the dynamic equilibrium between new immigrated species onto an island and the extinct species out from that island $[2,3,8]$.

Fig. 1 graphically represents the equilibrium model with linear immigration (or speciation) rate $\lambda$ and emigration (or extinction) rate $\mu$, where they can be plotted as logistic, exponential or any proper function $[4,10,11]$.

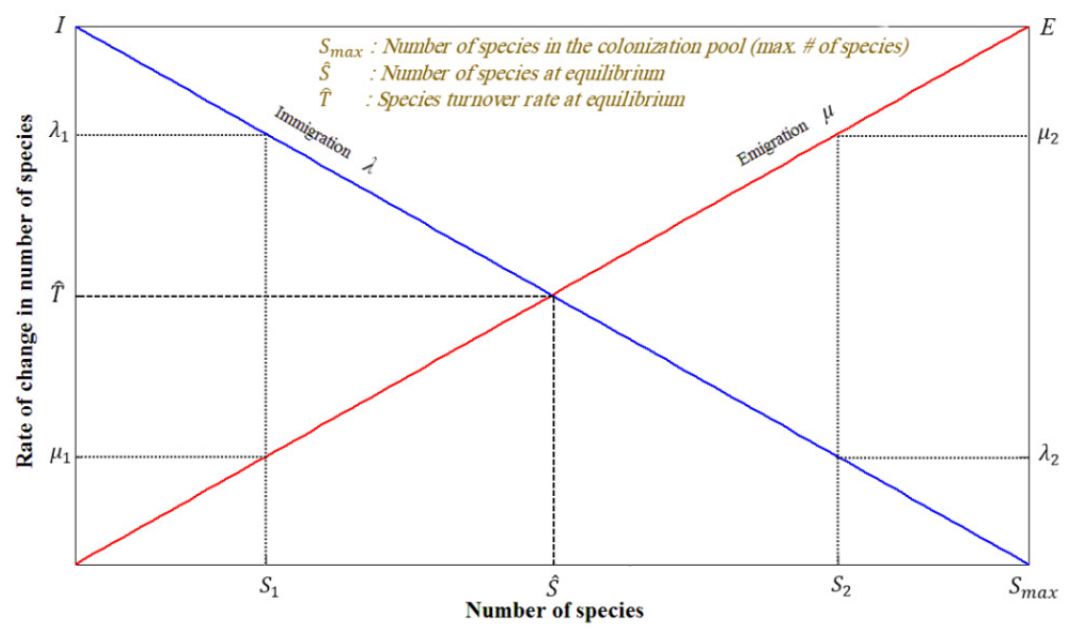

Figure 1. Simplified equilibrium model of a biota of a single island

$I$ and $E$ are the maximum possible immigration and emigration rates, respectively. Ioccurs when the island is empty of any species and thus it offers a maximum opportunity to the species on the other islands for immigrating to settle on it; whereas the arrivals on that island increases, the 
opportunity for settlement will decrease, which means that the immigration rate decreases. Also, as $\lambda$ decreases, the species density increases, and thus the predation, competition and parasitism factors will increase too; and as a result, the emigration rate $\mu$ will increase and reaches its maximum value $E$ when $\lambda$ reaches its minimum value [12].

MacArthur and Wilson, in their early study [2,3], proposed a simplified equilibrium model with $I=E$, where at time $t$, the recipient island has $S$ species with probability $P_{s}(t)$, and $\lambda_{s}$ and $\mu_{s}$ are the immigration and emigration rates at the present of $S$ species in that island.Then the variation from $P_{S}(t)$ to $P_{S}(t+\Delta t)$ can be described as:

$$
P_{S}(t+\Delta t)=P_{S}(t)\left(1-\lambda_{s} \Delta t-\mu_{s} \Delta t\right)+P_{s-1}(t) \lambda_{s-1} \Delta t+P_{s+1}(t) \mu_{s+1} \Delta t
$$

From Eq. 1, to have $S$ at time $(t+\Delta t)$, one of the following three conditions should hold:

1. $S$ species at time $t$, and no immigration or emigration took place during the interval $\Delta t$;

2. $(S-1)$ species at time $t$, and one species immigrated;

3. $(S+1)$ species at time $t$, and one species emigrated.

The probability of immigration and/or emigration should not be more than one, and thus $\Delta t$ has to be set with small value. Now, as $\Delta t$ approaches 0 , the ratio $\left(\frac{\Delta P_{S}}{\Delta t}\right)$ approaches $\dot{P}_{S}(t)$ :

$$
\begin{aligned}
& \frac{d P_{S}(t)}{d t} \cong \lim _{\Delta t \rightarrow 0} \frac{P_{S}(t+\Delta t)-P_{S}(t)}{\Delta t} \\
& \frac{d P_{S}(t)}{d t} \cong-\left(\lambda_{s}+\mu_{S}\right) P_{S}(t)+\lambda_{s-1} P_{S-1}(t)+\mu_{s+1} P_{S+1}(t)
\end{aligned}
$$

By considering the above three conditions, Eq. 2 can be specified more as:

$$
\dot{P}_{S}(t)=\left\{\begin{array}{cc}
-\left(\lambda_{s}+\mu_{s}\right) P_{s}+\mu_{s+1} P_{s+1}, & S=0 \\
-\left(\lambda_{s}+\mu_{s}\right) P_{s}+\lambda_{s-1} P_{s-1}+\mu_{s+1} P_{s+1}, & 1 \leq S \leq S_{\text {max }}-1 \\
-\left(\lambda_{s}+\mu_{s}\right) P_{s}+\lambda_{s-1} P_{s-1}, & S=S_{\max }
\end{array}\right.
$$

If $P_{S}(t)$ is known, then $\dot{P}_{S}(t)$ can be obtained from Eq. 3, where the value of $P_{S}(t+\Delta t)$ in Eq. 1 can be approximated as:

$$
P_{S}(t+\Delta t) \cong P_{S}(t)+\dot{P}_{S}(t) \Delta t
$$

Eq. 4 is the final confirmed form that has to be used in the program of $\mathrm{BBO}$ for calculating $P_{s}(t+$ $\Delta t)$.

For finding $P_{S}(t)$, Dan Simon in [1] gives two methods; either by solving Eq. 3 numerically, or applying the following theorem:

Theorem 1: The steady-state value for the probability of the number of each species is given by:

$$
P(\infty)=\frac{v}{\sum_{i=1}^{s_{\max }+1} v_{i}}
$$

Where $v$ and $v_{i}$ can be computed from the following eqs.: 


$$
\begin{gathered}
v=\left[v_{1}, v_{2}, \ldots, v_{S_{\max }+1}\right]^{T} \\
v_{i}=\frac{S_{\max } !}{\left(S_{\max }+1-i\right) !(i-1) !} \quad\left(i=1, \ldots, S_{\max }+1\right)
\end{gathered}
$$

Although the second alternative is easier and $P_{S}(t)$ can be computed directly without any iteration, this method is not preferable in the most programs, such as $\mathrm{C} / \mathrm{C}++$, MATLAB, Octave, Maple, Python, etc, because it is valid only when $S_{\max } \leq 170$, otherwise $S_{\max }$ ! $=\infty$, unless an additional sub-algorithm is used to overcome this problem. In addition, this approach will consume extra CPU time for dealing with long product operations.

The remaining terms for finding $P_{s}(t+\Delta t)$ are $\mu_{s}$ and $\lambda_{s}$, which can be calculated directly as:

$$
\begin{gathered}
\mu_{s}=\frac{E}{S_{\max }} S \\
\lambda_{s}=1-\mu_{s}=I\left(1-\frac{S}{S_{\max }}\right)
\end{gathered}
$$

\section{BIOGEOGRAPHY-BASED OPTIMIZATION (BBO)}

BBO translates the natural distribution of species into a general problem solution [1]. Each island represents one solution, where the good problem solution means that the island has lots of good biotic "living: diversity of prey, trees, shrubs, meadow, etc" and abiotic "non-living: distance of isolation, wind, temperature, humidity, water, area, etc" factors, which attracts more species than the other islands [4]. Each featureis called suitability index variable (SIV), which represents the independent variable of such a problem in BBO. As these features changes, the island suitability index (ISI) changes too; thus in BBO, ISIis the dependent variable $[1,17]$.

A problem with $n$-independent variables and $k$-islands or individuals can be expressed as:

$$
I S I_{i}=f\left(S I V_{1}, S I V_{2}, \ldots, S I V_{n}\right) \quad i=1,2, \ldots, k
$$

In the early stages of introducing BBO, Dan Simon proposed four different types of migration process, these types can be sorted as [15]:

1. Partial Migration Based BBO "PMB-BBO"

2. Single Migration Based BBO "SMB-BBO"

3. Simplified Partial Migration Based BBO "SPMB-BBO"

4. Simplified Single Migration Based BBO "SSMB-BBO"

From preceding study [20], it is shown that SMB-BBO and SSMB-BBO give poor performance but with lowest CPU time, while the performance comparison between PMB-BBO and SPMB$\mathrm{BBO}$ shows that $\mathrm{PMB}-\mathrm{BBO}$ gives better performance as the complexity, side constrains and/or dimensions of a given problem increases and as the number of islands decreases; and vice versa for SPMB-BBO.

In general, SPMB-BBO could trap in a local or at least a near-global optima, especially when the mutation stage that compensate the weakness of its migration algorithm is absent. This drawback led us to select PMB-BBO as a final confirmed BBO model for applying the proposed essential modification, which will be explained in the next section. 
The algorithm of BBO consists of two main stages, migration and mutation.

\subsection{Migration}

Considering Fig. 1 and Eq. 10, if island $i$ has lots of features, then lots of species will colonize it, which means that $\lambda_{s}$ becomes low and $\mu_{s}$ becomes high.

Thus, the high ISI for island irepresents a good solution, and vice versa for a poor solution which has a shortage in its features diversity, and reflected on the total available number of species; where at this condition, $\lambda_{s}$ is high and $\mu_{s}$ is low.

From Fig. 1, $S_{l}$ is located before $\hat{S}$, where $\lambda_{s}$ is high, $\mu_{S}$ is low and the solution $I S I_{l}$ is poor; while $S_{2}$ is located after $\hat{S}$, where $\lambda_{s}$ is low, $\mu_{s}$ is high and the solution $I S I_{2}$ is good. Based on that, $\lambda_{s}$ and $\mu_{s}$ can be used as indications of poor and good solutions, respectively.

The purpose of migration process is to use high ISI islands as a source of modification to share their features with low ISI islands, so the poor solutions can be probabilistically enhanced and may become better than those good solutions.

The migration process of PMB-BBO can be described as:

Let $I S I_{i}$ denote the $i$ th population member and contains $n$ features

For each island $I S I_{i}$ (where $i=1,2,3, \ldots, k$ )

For each $S I V s$ (where $s=1,2,3, \ldots, n$ )

Use $\lambda_{i}$ to probabilistically select the immigrating island $I S I_{i}$

If rand $<\lambda_{i}$

For $j=1$ to $k$

Use $\mu_{j}$ to probabilistically decide whether to emigrate to $I S I_{i}$

If $I S I_{j}$ is selected

Randomly select an $S I V \sigma$ from $I S I_{j}$

Replace a random $S I V s$ in $I S I_{i}$ with $S I V \sigma$

end if

end for

end if

nextSIV

next island

\subsection{Mutation}

In island theory, the species at equilibrium point $\hat{S}$ can be deviated dramatically due to some external events. Events such as predators from other islands, tsunamis, volcanos, diseases or earthquakes cause negative deviation, and the total number of species will steeply decreases [11]. On the other hand, there are some other useful events such as wind-carrying seeds or flotsams 
which provide good features to an island, thus giving better solution with a significant enhancement [12].In BBO, the mutation process is modeled as SIV mutation; and through species count probabilities $P_{s}$, the mutation rate $m$ can be determined as:

$$
m=m_{\max }\left(1-\frac{P_{s}}{P_{\max }}\right)
$$

$m_{\max }$ is a user-defined maximum mutation rate that $m$ can reach, and $P_{\max }=\max \left(P_{s}\right)$.

From the previous equation, $m$ reaches to its minimum "zero" at the maximum value of $P_{s}$, and vice versa. Thus, $m$ is inversely proportional to $P_{s}$. This process can be graphically described as in Fig. 2, where the species count $S$ starts from zero to $S_{\max }$. As $m_{\max }$ increases, the chance to let the solutions be mutated increases too.

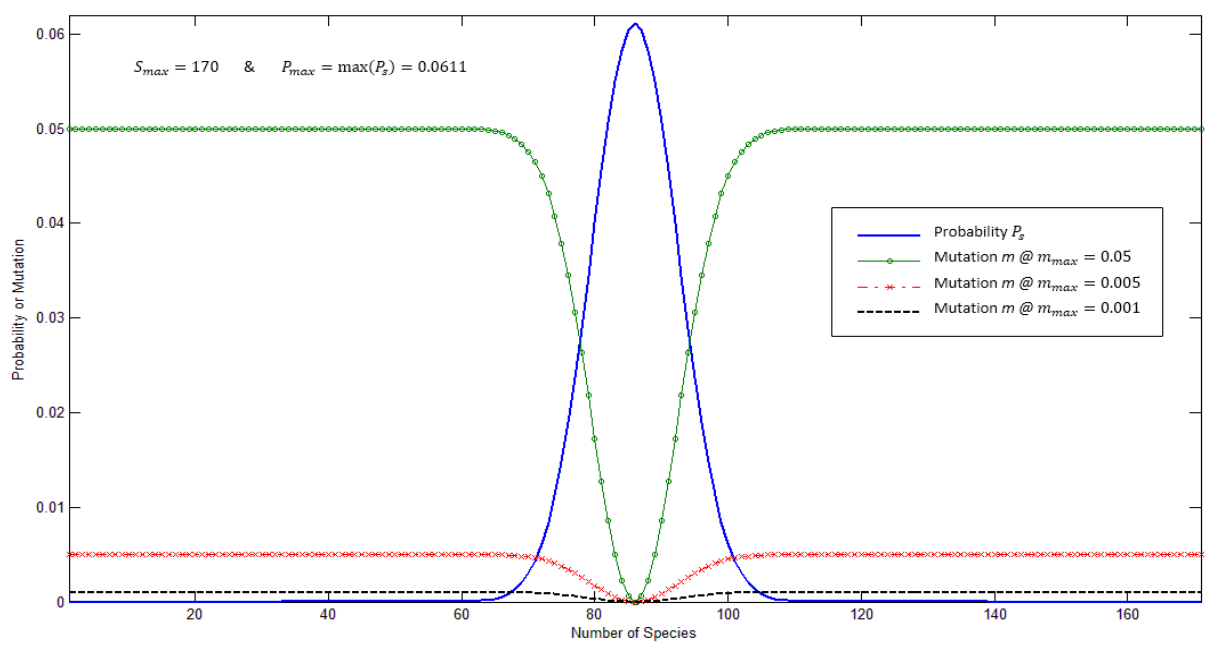

Figure 2. Comparison between $P_{s}(t)$ and $m(t)$ at different $m_{\max }$

During the mutation stage, the low and high ISI solutions are likely to mutate, and then could be enhanced more than what they already have, where the solutions at the equilibrium point are not mutated [1]. Even if the mutated solutions become worse, the optional stage, called elitism, will store the best solutions from one generation to the next [17].

The mutation process can be described as:

For $i=1$ to $k$ (where $\mathrm{k}$ is the number of islands, see Eq. 10)

Calculate probability $P_{s}$ based on $\lambda_{s}$ and $\mu_{s}$ (by numerical or direct method)

Calculate mutation rate $m$ (using Eq. 11)

Select $I S I_{i}$ with probability proportional to $P_{s}$

If $I S I_{i}$ is selected

Replace $S I V$ of $I S I_{i}$ with a randomly generated $S I V$

end if

end for 


\subsection{BBO Algorithm}

The BBO algorithm can be summarized through the flowchart of Fig.3.

The algorithm's looping can be terminated either if reaches to an acceptable tolerance or after completing the desired number of generations.

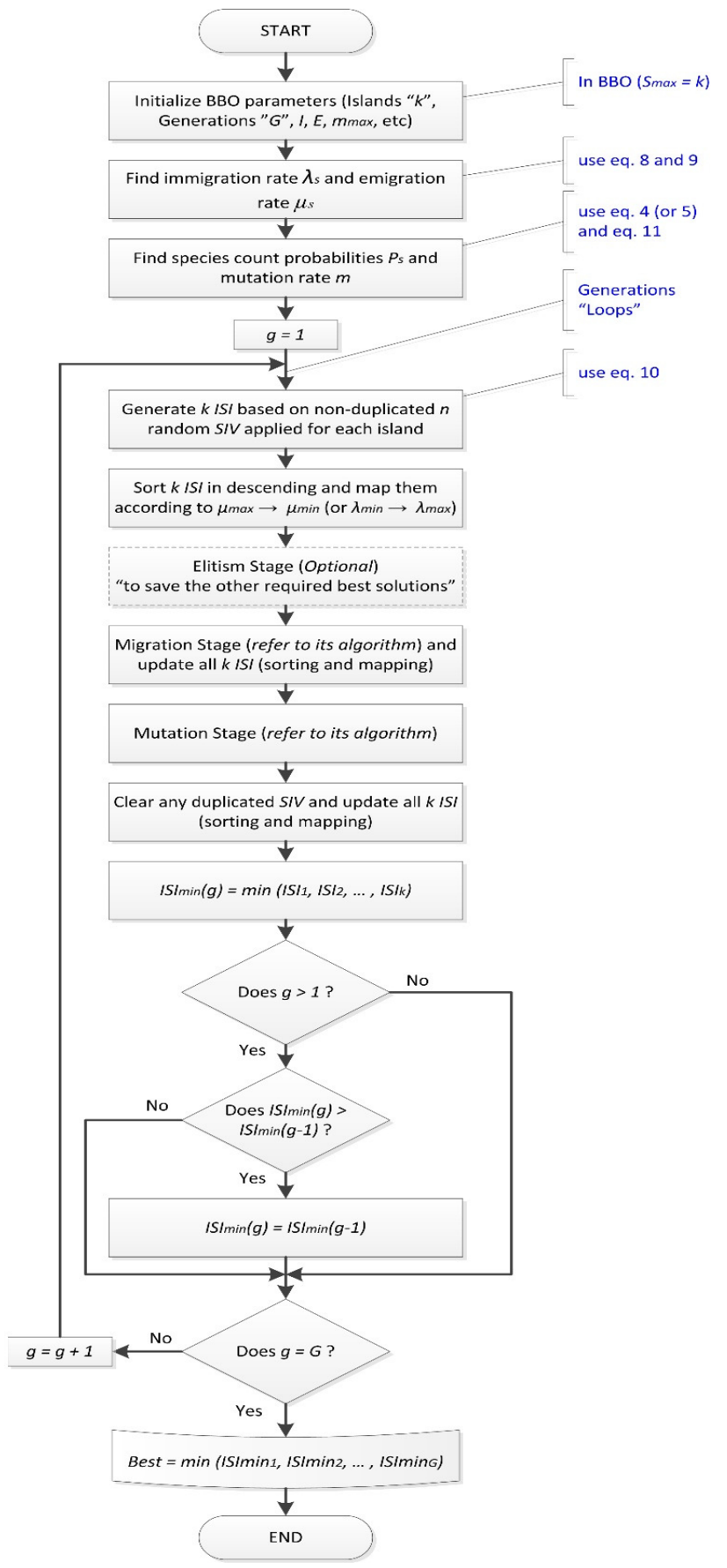

Figure 3. General flowchart of BBO algorithm 


\section{The Proposed Essential Modification on BBO}

This paper tries to solve two fundamental problems that are associated with the original version or the raw form of $\mathrm{BBO}$ with using just a linear immigration and emigration rates.

By accomplishing this modification, it can be used a basis for any further modification or hybridization. This study shows that the linear immigration and emigration rates can do well if the associated root problems are solved.

According to the original BBO program that was designed by Dan Simon in [21], there are two essential parts that need to be solved:

\subsection{Probabilistic Selection Process of the Migration and Mutation Stages}

Referring to the preceding algorithms, the process for selecting SIVs of an island $i$ that needs to be migrated is done probabilistically, and the general code for this task is:

$$
\operatorname{SIV}_{S}(1: k)=\operatorname{round}[1+(n-1) \times \operatorname{rand}(1, n)]
$$

If the independent variables of each $I S I$ are represented as a vector of $\left[S I V_{1}, S I V_{2}, \ldots, S I V_{n}\right]$, then the analysis of the above code shows that the SIVs at the beginning and at the end have less weight than the other (n-2) SIV that are located in between.

Fig. 4a shows how unfairly selection be done for each $S I V$ of island $i$. In this example; $n=5$ and $k=1000$, with 4 trails.

In MATLAB, the proposed modification is to use integer random "randi" instead of using rounded real random "rand" with $n S I V$ alignment, as in Eq. 12.This integer random function provides pseudorandom integers from a uniform discrete distribution on 1to $n$.

For getting integer random values with fairly selection for all $n S I V$ :

$$
\operatorname{SIV}_{S}(1: k)=\operatorname{randi}(n, 1, n)
$$

This MATLAB code given in Eq. 13 is equivalent to the previous code in Eq. 12, but with significant enhancement. The same analysis is done for this code, and the result is shown in Fig. $4 \mathrm{~b}$. It is clearly seen that the selection process for the migrated and mutated $n S I V$ is enhanced.

Note that, this part of modification is not available for 1-dimensional problems, and has less effect for 2-dimensional problems.

\subsection{Clear Duplication Process of the Mutation Stage}

According to the original BBO [21], only the worst solutions are mutated. The range of these mutated solutions can be defined through the preceding mutation algorithm in Section 3.2 as:

For $m=$ round $\left[\right.$ length $\left(\frac{\text { Islands }}{d}\right):$ length(Islands $\left.)\right]$

Do mutation (refer to its algorithm) end for 
If $d=2$, then the worst half of the solutions are to be mutated; and as $d$ increases, the percentage of the total mutated solutions increases too.
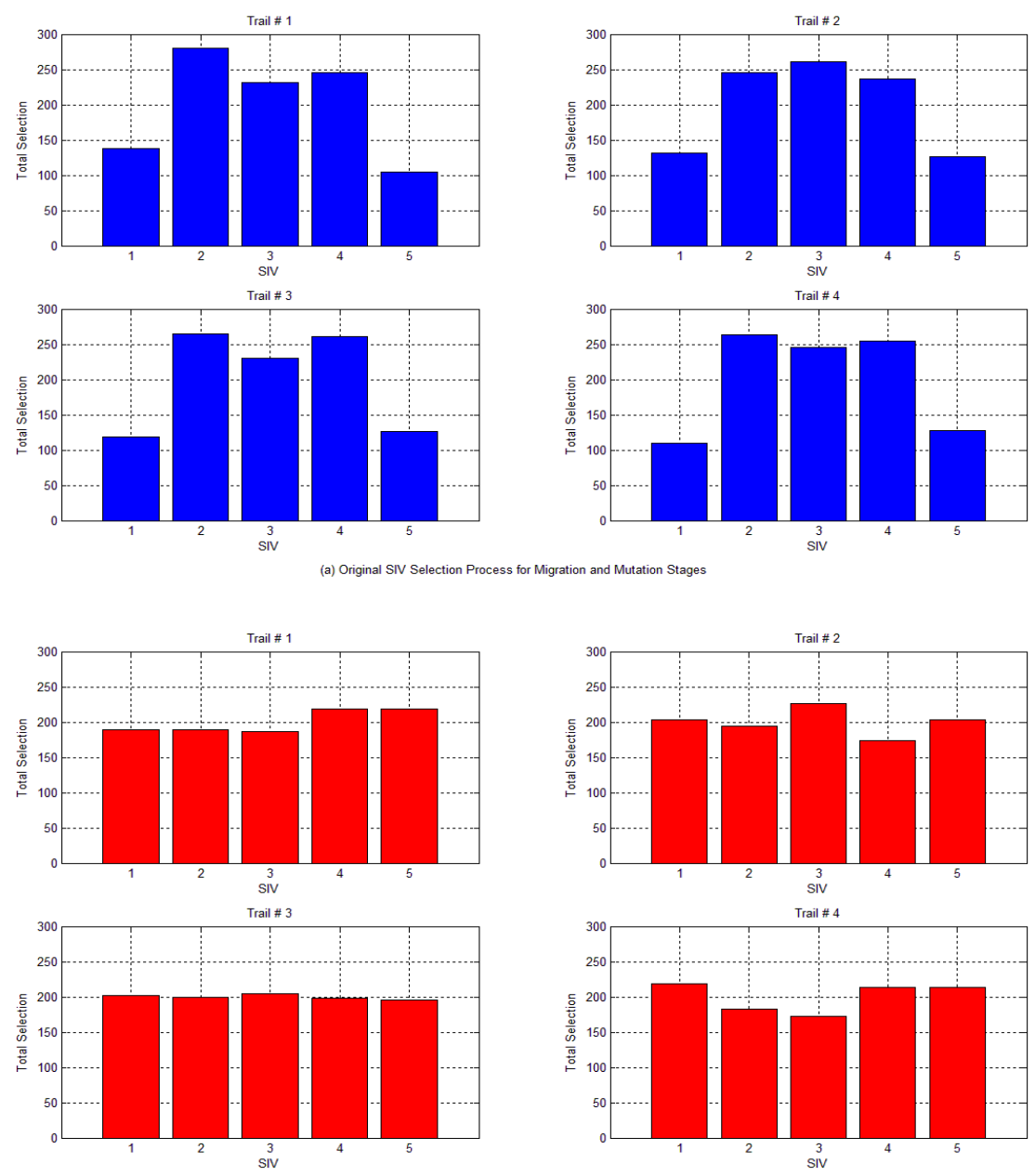

Figure 4. The Original and Modified SIV-Selection Process for Migration and Mutation Stages

The modification of this part is to let the clear duplication process be done only on the mutated solutions, so that the $n S I V$ of the non-mutated solutions are kept away from any change. Without this modification, the migrated features of those non-mutated islands will be corrupted.

\section{Performance Comparison}

The original and modified versions of PMB-BBO have been tested through a wide variety range of test functions with different dimensions and complexities, where all the details of each test function are given in the Appendix.

Table 1a shows the parameters used for both BBOs. These parameters are similar to those used in $[13,16]$, but with more restriction on the generation limits, which are listed in Table $1 b$. 
Table 1a. BBOs' parameters (For more details refer to $[13,16]$ )

\begin{tabular}{|c|c|}
\hline Parameter & Value \\
\hline Population size - or $k$ & 50 \\
\hline Max. $\lambda-$ or $I$ & 1 \\
\hline Max. $\mu-$ or $E$ & 1 \\
\hline$m_{\max }$ & 0.01 \\
\hline Elitism & 1 \\
\hline Mutation range & round $\left[\left(\frac{k}{2}\right): k\right]$ \\
\hline Number of trails & 30 \\
\hline
\end{tabular}

Table 1b. Required generations for various n-dimensional problems

\begin{tabular}{|c|c|}
\hline Problem's dimension & \# of generations \\
\hline $1,2,3,4,5$ or 6 & 1,000 \\
\hline 8,9 or 10 & 5,000 \\
\hline 15,17 or 20 & 10,000 \\
\hline 30 & 20,000 \\
\hline 60 & 50,000 \\
\hline
\end{tabular}

The performance comparison are evaluated using 120 test functions. They are split into two equally groups; the first group contains only 2-dimensional test functions and are listed in Table $2 \mathrm{a}$, while the second group contains other $\mathrm{n}$-dimensional test functions and are listed in Table $2 \mathrm{~b}$. The reason for this arrangement is because this paper is a part of a project that deals with 2dimensional engineering problem, and the obtained result from this paper will be used a basis to determine if this essential modification gives a better performance or not before implementing it for solving that particular problem.

Therefore, more effort was done on 2-dimensional test functions to cover a gradient of difficulties of unimodal and multimodal functions in conjunction with few and many local minima as traps, where the diversity of variable bounds through narrow and wide search spaces provides other challenges.

Table $2 \mathrm{a}$ and Table $2 \mathrm{~b}$ give the best, mean and standard deviation of the 120 test functions. As an overall, the obtained results shows that the performance of PMB-BBO can be enhanced effectively by applying the proposed essential modification on its migration and mutation stages with resizing the range of clear duplication process.

However, for 1-dimensional problems, this proposed modification is not effective because they have only one $S I V$, which means that the migration and mutation stages are processed within one independent variable. Thus, the first part of the proposed modification is absent. Based on this study, this new version of PMB-BBO can do well if both parts of the modification are embedded. This can be seen from the first eight test functions shown in Table 2b. Similarly, for other ndimensional problems (where $n \geq 2$ ), the modified PMB-BBO shows better performance and wins in most test functions. From the results of the best error, the mean, and the standard deviation, it can concluded that the proposed correction on the randomized selection process of the migration and mutation algorithms improves its exploration and exploitation. Fig. 5 shows the curves of fitness functions of both versions for the Generalized Rastrigin's function, Schwefel's problem 1.2, Qing's function and Salomon's function. It can be clearly seen that the modified PMB-BBO can converge to a better solution more than the original version.

The modified PMB-BBO has proved that this essential modification is highly recommended for problems with dimension higher than 1 . Even, for the few test functions, where the original version shows better results, the modified version gives a competitive results. 
Table 2a. Comparison of results over 30 trails of the original and modified versions of PMB-BBO, where "Best" means the best error, "Mean" indicates the mean best error, and "StdDev" stands for the standard deviation - It contains just 2-dimensional test functions

\begin{tabular}{|c|c|c|c|c|c|c|c|c|}
\hline \multirow{3}{*}{ Func. \# } & \multirow{3}{*}{ Function Name } & \multirow{3}{*}{$\mathbf{n}$} & \multicolumn{6}{|c|}{ Biogeography Based Optimization (BBO) } \\
\hline & & & \multicolumn{3}{|c|}{ Original Partial Migration Based } & \multicolumn{3}{|c|}{ Modified Partial Migration Based } \\
\hline & & & Best & Mean & StdDev & Best & Mean & StdDev \\
\hline f2-01 & Aluffi-Pentini & 2 & $1.4073 \mathrm{E}-07$ & $4.3166 \mathrm{E}-05$ & $5.3028 \mathrm{E}-05$ & $1.1278 \mathrm{E}-07$ & $8.8268 \mathrm{E}-06$ & $1.0718 \mathrm{E}-05$ \\
\hline f2-02 & Banana Shape & 2 & 3.2053E-07 & $3.4468 \mathrm{E}-04$ & $5.4340 \mathrm{E}-04$ & $2.9272 \mathrm{E}-06$ & 7.4032E-04 & $8.3409 \mathrm{E}-04$ \\
\hline f2-03 & Beale & 2 & \begin{tabular}{|l|}
$2.8187 E-06$ \\
\end{tabular} & $1.6885 \mathrm{E}-04$ & $1.6853 \mathrm{E}-04$ & \begin{tabular}{|l|}
$3.0033 \mathrm{E}-06$ \\
\end{tabular} & 3.5367E-04 & $5.3948 \mathrm{E}-04$ \\
\hline f2-04 & Becker-Lago & 2 & 9.1117E-08 & $1.4622 \mathrm{E}-05$ & $1.7747 \mathrm{E}-05$ & \begin{tabular}{|l|}
$1.6084 \mathrm{E}-08$ \\
\end{tabular} & $3.5743 \mathrm{E}-06$ & 6.8117E-06 \\
\hline f2-05 & Bird & 2 & $7.0155 \mathrm{E}-07$ & $2.8262 \mathrm{E}-03$ & 2.8437E-03 & $1.2209 \mathrm{E}-05$ & 3.5197E-04 & 4.4087E-04 \\
\hline f2-06 & Bohachevsky F1 & 2 & $2.6931 \mathrm{E}-05$ & $1.1511 \mathrm{E}-03$ & $1.2958 \mathrm{E}-03$ & \begin{tabular}{|l|}
$8.4775 E-07$ \\
\end{tabular} & $2.4441 \mathrm{E}-04$ & $3.2364 \mathrm{E}-04$ \\
\hline $\mathrm{f2}-07$ & Bohachevsky F2 & 2 & \begin{tabular}{|l|}
$5.2095 \mathrm{E}-06$ \\
\end{tabular} & $1.5371 \mathrm{E}-03$ & $1.7717 \mathrm{E}-03$ & \begin{tabular}{|c|}
$1.5425 \mathrm{E}-06$ \\
\end{tabular} & \begin{tabular}{|l|}
$1.2837 \mathrm{E}-04$ \\
\end{tabular} & $1.6563 \mathrm{E}-04$ \\
\hline f2-08 & Bohachevsky F3 & 2 & 9.8338E-05 & $2.5948 \mathrm{E}-03$ & $4.0705 \mathrm{E}-03$ & $1.8607 E-05$ & 3.4955E-03 & $4.0741 \mathrm{E}-03$ \\
\hline f2-09 & Booth & 2 & \begin{tabular}{|l|}
$2.3450 \mathrm{E}-05$ \\
\end{tabular} & $1.3871 \mathrm{E}-03$ & $1.3831 \mathrm{E}-03$ & \begin{tabular}{|l|}
$7.0317 E-08$ \\
\end{tabular} & $2.7318 \mathrm{E}-04$ & 3.8789E-04 \\
\hline f2-10 & Branin RCOS & 2 & \begin{tabular}{|l}
$5.6480 \mathrm{E}-07$ \\
\end{tabular} & 9.6466E-05 & 1.6743E-04 & \begin{tabular}{|l}
$6.1344 \mathrm{E}-07$ \\
\end{tabular} & \begin{tabular}{|l}
$5.2541 \mathrm{E}-05$ \\
\end{tabular} & \begin{tabular}{|l}
$1.3086 \mathrm{E}-04$ \\
\end{tabular} \\
\hline f2-11 & Bukin F4 & 2 & \begin{tabular}{|l|}
$7.9693 \mathrm{E}-07$ \\
\end{tabular} & 6.6953E-05 & $6.2600 \mathrm{E}-05$ & \begin{tabular}{|c|}
$9.5554 \mathrm{E}-07$ \\
\end{tabular} & 4.1762E-05 & $5.5448 \mathrm{E}-05$ \\
\hline $\mathrm{f} 2-12$ & Bukin F6 & 2 & 1.4263E-01 & 5.5443E- 01 & 2.3743E- 01 & 1.2043E- 01 & $6.5545 \mathrm{E}-01$ & 3.2602E-01 \\
\hline f2-13 & Carrom Table & 2 & 1.6143E-06 & 2.6772E-04 & 3.3997E-04 & 4.6210E- 08 & \begin{tabular}{|l|}
$5.5445 \mathrm{E}-05$ \\
\end{tabular} & \begin{tabular}{|l}
$5.1728 \mathrm{E}-05$ \\
\end{tabular} \\
\hline f2-14 & Chichinadze & 2 & 4.2929E-06 & $8.3095 \mathrm{E}-03$ & $1.6882 \mathrm{E}-02$ & $3.6249 \mathrm{E}-06$ & $4.4121 \mathrm{E}-03$ & $6.0434 \mathrm{E}-03$ \\
\hline f2-15 & Complex & 2 & \begin{tabular}{|l|}
$3.3448 \mathrm{E}-09$ \\
\end{tabular} & 1.1847E-05 & $1.4765 \mathrm{E}-05$ & $1.1044 \mathrm{E}-08$ & 2.4003E-06 & $3.0318 \mathrm{E}-06$ \\
\hline f2-16 & Cosine Mixture & 2 & \begin{tabular}{|l|}
$6.6629 \mathrm{E}-08$ \\
\end{tabular} & 4.9456E-06 & 5.9989E-06 & $1.7583 \mathrm{E}-11$ & 1.2499E-06 & $2.0690 \mathrm{E}-06$ \\
\hline f2-17 & Cross In Tray & 2 & \begin{tabular}{|l|}
$1.6206 \mathrm{E}-08$ \\
\end{tabular} & 3.1863E-06 & $4.7941 \mathrm{E}-06$ & \begin{tabular}{|l|}
$5.1076 \mathrm{E}-09$ \\
\end{tabular} & $5.2181 \mathrm{E}-07$ & 5.9861E-07 \\
\hline $\mathrm{f} 2-18$ & Cross Leg Table & 2 & \begin{tabular}{|l|}
$9.9939 \mathrm{E}-01$ \\
\end{tabular} & 9.9959E-01 & $8.6652 \mathrm{E}-05$ & \begin{tabular}{|c|}
$9.9933 \mathrm{E}-01$ \\
\end{tabular} & \begin{tabular}{|c|}
$9.9960 \mathrm{E}-01$ \\
\end{tabular} & 9.1172E-05 \\
\hline f2-19 & Crowned Cross & 2 & 1.2851E-01 & 2.6119E-01 & \begin{tabular}{|l|}
$3.8380 \mathrm{E}-02$ \\
\end{tabular} & $1.5692 \mathrm{E}-01$ & \begin{tabular}{|l|}
$2.4251 \mathrm{E}-01$ \\
\end{tabular} & 4.1245E-02 \\
\hline $\mathrm{f} 2-20$ & Davis & 2 & \begin{tabular}{|l|}
$1.4899 \mathrm{E}-01$ \\
\end{tabular} & 4.0370E-01 & $1.3762 \mathrm{E}-01$ & \begin{tabular}{|l|}
$8.7947 E-02$ \\
\end{tabular} & $2.6525 \mathrm{E}-01$ & $1.1171 \mathrm{E}-01$ \\
\hline $\mathrm{f} 2-21$ & Decanomial & 2 & \begin{tabular}{|l|}
$2.3936 \mathrm{E}-06$ \\
\end{tabular} & 1.2007E-02 & $2.0762 \mathrm{E}-02$ & \begin{tabular}{|l|}
$3.6446 \mathrm{E}-05$ \\
\end{tabular} & 3.0835E-02 & $8.4008 \mathrm{E}-02$ \\
\hline f2-22 & Dekkers-Aarts & 2 & 4.9519E-01 & $2.6947 \mathrm{E}+00$ & $3.6355 \mathrm{E}+00$ & $4.8362 \mathrm{E}-01$ & $1.8882 \mathrm{E}+00$ & $3.5944 \mathrm{E}+00$ \\
\hline f2-23 & Drop Wave & 2 & 6.5366E- 06 & 3.6741E-03 & \begin{tabular}{|l|}
$1.1802 \mathrm{E}-02$ \\
\end{tabular} & \begin{tabular}{|l}
$1.7502 \mathrm{E}-05$ \\
\end{tabular} & 7.8462E-03 & 1.9646E-02 \\
\hline f2-24 & Easom & 2 & 3.3330E-06 & 1.0643E-04 & $8.4788 \mathrm{E}-05$ & $8.7507 E-08$ & \begin{tabular}{|l|}
$1.8423 \mathrm{E}-05$ \\
\end{tabular} & 3.1394E-05 \\
\hline f2-25 & Egg Holder & 2 & $8.1682 \mathrm{E}-02$ & $4.4751 \mathrm{E}+00$ & $2.5844 \mathrm{E}+00$ & 4.1432E- 02 & $3.6928 \mathrm{E}+00$ & $5.8969 \mathrm{E}+00$ \\
\hline f2-26 & EXP2 & 2 & $1.3177 \mathrm{E}-07$ & $2.0891 \mathrm{E}-05$ & $3.0858 \mathrm{E}-05$ & 9.5422E- 08 & \begin{tabular}{|l|}
$4.4703 \mathrm{E}-06$ \\
\end{tabular} & 6.1713E-06 \\
\hline f2-27 & Freudenstein-Roth & 2 & \begin{tabular}{|l|}
$5.4859 \mathrm{E}-05$ \\
\end{tabular} & $1.0514 \mathrm{E}-02$ & $2.1059 \mathrm{E}-02$ & \begin{tabular}{|l|}
$2.0160 \mathrm{E}-06$ \\
\end{tabular} & 4.2978E-03 & 6.6492E- 03 \\
\hline $\mathrm{f2}-28$ & Giunta & 2 & $2.4751 \mathrm{E}-08$ & 5.0673E-07 & $6.3542 \mathrm{E}-07$ & \begin{tabular}{|l|}
$7.6581 \mathrm{E}-10$ \\
\end{tabular} & \begin{tabular}{|l|}
$8.1474 \mathrm{E}-08$ \\
\end{tabular} & $1.1653 \mathrm{E}-07$ \\
\hline f2-29 & Goldstein-Price & 2 & \begin{tabular}{|l|}
$2.7778 \mathrm{E}-05$ \\
\end{tabular} & $1.5749 \mathrm{E}-03$ & $1.6278 \mathrm{E}-03$ & \begin{tabular}{|l|}
$3.1758 \mathrm{E}-06$ \\
\end{tabular} & 2.7174E-04 & 4.1521E-04 \\
\hline $\mathrm{f} 2-30$ & Himmelblau & 2 & \begin{tabular}{|l|}
$5.9826 \mathrm{E}-06$ \\
\end{tabular} & 9.4920E-04 & $1.2093 \mathrm{E}-03$ & \begin{tabular}{|l|}
$1.4971 E-06$ \\
\end{tabular} & \begin{tabular}{|l|}
$7.2058 \mathrm{E}-05$ \\
\end{tabular} & $9.5358 \mathrm{E}-05$ \\
\hline f2-31 & Holder Table & 2 & \begin{tabular}{|l|}
$6.7954 \mathrm{E}-07$ \\
\end{tabular} & 7.5566E-05 & 1.2147E-04 & \begin{tabular}{|l|}
$6.7237 \mathrm{E}-07$ \\
\end{tabular} & 1.6798E-05 & $2.3888 \mathrm{E}-05$ \\
\hline $\mathrm{f} 2-32$ & Hosaki & 2 & 2.9862E-08 & $1.2363 \mathrm{E}-05$ & $1.3592 \mathrm{E}-05$ & $1.9118 \mathrm{E}-08$ & \begin{tabular}{|l|}
$2.2394 \mathrm{E}-06$ \\
\end{tabular} & 2.3016E-06 \\
\hline f2-33 & Kearfott & 2 & $1.4138 \mathrm{E}-06$ & $1.7270 \mathrm{E}-05$ & $2.3710 \mathrm{E}-05$ & \begin{tabular}{|c|}
$9.0621 \mathrm{E}-09$ \\
\end{tabular} & 3.6849E-06 & 6.8312E-06 \\
\hline$f 2-34$ & Inverted Cosine Wave & 2 & \begin{tabular}{|l}
$8.6479 \mathrm{E}-06$ \\
\end{tabular} & 5.0150E-04 & 5.6270E-04 & \begin{tabular}{|l|}
$2.5292 E-06$ \\
\end{tabular} & 4.7085E-05 & $1.0482 \mathrm{E}-04$ \\
\hline $\mathrm{f} 2-35$ & Levy F3 (or Hansen) & 2 & 3.4917E-04 & $1.9752 \mathrm{E}-02$ & 2.4033E-02 & \begin{tabular}{|l|}
$2.1775 E-04$ \\
\end{tabular} & $1.7541 \mathrm{E}-03$ & 2.2954E-03 \\
\hline $\mathrm{f} 2-36$ & Levy F5 & 2 & 5.5062E-05 & $1.4691 \mathrm{E}-01$ & $1.4975 \mathrm{E}-01$ & 6.4056E-05 & \begin{tabular}{|l|}
$2.2562 \mathrm{E}-02$ \\
\end{tabular} & 2.5591E-02 \\
\hline $\mathrm{f} 2-37$ & Matyas & 2 & 2.5988E-07 & 6.4073E-05 & $7.3529 \mathrm{E}-05$ & 5.2695E- 07 & \begin{tabular}{|l|}
$3.8882 \mathrm{E}-05$ \\
\end{tabular} & 4.2294E-05 \\
\hline $\mathrm{f} 2-38$ & McCormick & 2 & $1.9661 \mathrm{E}-07$ & $1.9014 \mathrm{E}-05$ & $2.7088 \mathrm{E}-05$ & 9.8424E- 08 & \begin{tabular}{|l|}
$3.2686 \mathrm{E}-06$ \\
\end{tabular} & 4.4669E-06 \\
\hline $\mathrm{f} 2-39$ & Michalewicz & 2 & \begin{tabular}{|l|}
$4.4897 \mathrm{E}-07$ \\
\end{tabular} & $1.4864 \mathrm{E}-05$ & 3.5212E-05 & $9.5133 \mathrm{E}-09$ & 3.6163E-06 & 5.3882E-06 \\
\hline f2-40 & Muller-Brown Surface & 2 & \begin{tabular}{|l|}
$8.2645 \mathrm{E}-04$ \\
\end{tabular} & $2.2639 \mathrm{E}-02$ & $2.2129 \mathrm{E}-02$ & $8.5253 \mathrm{E}-06$ & $8.2214 \mathrm{E}-03$ & $1.0327 \mathrm{E}-02$ \\
\hline $\mathrm{f2}-41$ & Parsopoulos & 2 & \begin{tabular}{|l|}
$3.3808 \mathrm{E}-12$ \\
\end{tabular} & 4.5059E-08 & 5.6394E-08 & \begin{tabular}{|l|}
$8.3716 \mathrm{E}-13$ \\
\end{tabular} & 2.2079E-08 & 2.7074E-08 \\
\hline $\mathrm{f} 2-42$ & Peaks & 2 & \begin{tabular}{|l|}
$7.0089 \mathrm{E}-07$ \\
\end{tabular} & $2.2400 \mathrm{E}-04$ & $2.7718 \mathrm{E}-04$ & \begin{tabular}{|l|}
$4.2985 \mathrm{E}-08$ \\
\end{tabular} & \begin{tabular}{|l|}
$3.7351 \mathrm{E}-05$ \\
\end{tabular} & $4.3122 \mathrm{E}-05$ \\
\hline $\mathrm{f} 2-43$ & Pen Holder & 2 & $8.5556 \mathrm{E}-09$ & $2.7320 \mathrm{E}-07$ & 4.6665E-07 & $1.9586 \mathrm{E}-10$ & \begin{tabular}{|l|}
$3.8556 \mathrm{E}-08$ \\
\end{tabular} & \begin{tabular}{|l}
$3.8049 \mathrm{E}-08$ \\
\end{tabular} \\
\hline $\mathrm{f} 2-44$ & Powell's Badly Scaled & 2 & \begin{tabular}{|l|}
$3.3630 \mathrm{E}-04$ \\
\end{tabular} & 6.4544E-01 & $3.7524 \mathrm{E}-01$ & \begin{tabular}{|c|}
$9.7414 \mathrm{E}-06$ \\
\end{tabular} & $8.9186 \mathrm{E}-01$ & 3.1950E-01 \\
\hline $\mathrm{f2}-45$ & Sawtoothxy & 2 & \begin{tabular}{|l|}
$7.1264 E-05$ \\
\end{tabular} & 3.2655E-03 & $6.8834 \mathrm{E}-03$ & 1.6672E-06 & \begin{tabular}{|l}
$3.1424 \mathrm{E}-04$ \\
\end{tabular} & 4.5476E-04 \\
\hline $\mathrm{f} 2-46$ & Schaffer's F1 & 2 & \begin{tabular}{|l|}
$9.5860 \mathrm{E}-04$ \\
\end{tabular} & $8.7166 \mathrm{E}-03$ & $2.4978 \mathrm{E}-03$ & $1.0451 \mathrm{E}-04$ & $9.2323 \mathrm{E}-03$ & $1.9361 \mathrm{E}-03$ \\
\hline $\mathrm{f} 2-47$ & Schaffer's F2 & 2 & $1.4803 \mathrm{E}+00$ & $6.6148 \mathrm{E}+00$ & $3.4823 \mathrm{E}+00$ & $1.3788 \mathrm{E}+00$ & $3.3010 \mathrm{E}+00$ & $1.2894 \mathrm{E}+00$ \\
\hline $\mathrm{f} 2-48$ & Shekel's Foxholes & 2 & 2.1720E-11 & $8.0558 \mathrm{E}-08$ & $2.1865 \mathrm{E}-07$ & 6.4642E-11 & \begin{tabular}{|l|}
$6.4142 \mathrm{E}-10$ \\
\end{tabular} & 5.9986E-10 \\
\hline $\mathrm{f} 2-49$ & Sinus oidal Problem & 2 & $2.2129 \mathrm{E}-07$ & 4.4691E-05 & $5.4481 \mathrm{E}-05$ & \begin{tabular}{|l|}
$2.6980 E-08$ \\
\end{tabular} & \begin{tabular}{|l|}
$3.1974 \mathrm{E}-06$ \\
\end{tabular} & 4.2395E-06 \\
\hline $\mathrm{f} 2-50$ & Stenger & 2 & $1.0055 \mathrm{E}-06$ & $1.7379 \mathrm{E}-04$ & 2.2777E-04 & 5.6936E- 07 & $6.4535 \mathrm{E}-05$ & 9.4907E-05 \\
\hline $\mathrm{f} 2-51$ & Storn & 2 & \begin{tabular}{|l|}
$3.9437 E-07$ \\
\end{tabular} & $1.3249 \mathrm{E}-06$ & $1.3478 \mathrm{E}-06$ & \begin{tabular}{|l|}
$3.9493 \mathrm{E}-07$ \\
\end{tabular} & $1.1236 \mathrm{E}-06$ & $1.2236 \mathrm{E}-06$ \\
\hline $\mathrm{f2}-52$ & Stretched V & 2 & $2.9346 \mathrm{E}-26$ & $1.4161 \mathrm{E}-16$ & 4.4560E-16 & 5.9429E-24 & $4.1241 \mathrm{E}-16$ & $1.3925 \mathrm{E}-15$ \\
\hline f2-53 & Test Tube Holder & 2 & \begin{tabular}{|l|}
$1.0791 \mathrm{E}-06$ \\
\end{tabular} & $8.0866 \mathrm{E}-05$ & 9.8752E-05 & \begin{tabular}{|l|}
$7.8935 E-08$ \\
\end{tabular} & 2.8773E-05 & $4.7662 \mathrm{E}-05$ \\
\hline $\mathrm{f2} 2-54$ & Treccani & 2 & 9.4387E-08 & $2.0880 \mathrm{E}-05$ & 2.7157E-05 & \begin{tabular}{|l|}
$3.8026 \mathrm{E}-08$ \\
\end{tabular} & 2.2693E-06 & $1.8565 \mathrm{E}-06$ \\
\hline $\mathrm{f2} 2-55$ & Trefethen F4 & 2 & \begin{tabular}{|l|}
$4.6587 \mathrm{E}-04$ \\
\end{tabular} & $9.5690 \mathrm{E}-02$ & $8.1159 \mathrm{E}-02$ & \begin{tabular}{|l|}
$9.1985 \mathrm{E}-04$ \\
\end{tabular} & 1.3107E-01 & $1.1657 \mathrm{E}-01$ \\
\hline $\mathrm{f} 2-56$ & Tripod & 2 & 5.2656E- 03 & $1.0925 \mathrm{E}-01$ & $6.3076 \mathrm{E}-02$ & 3.5156E- 03 & $5.3680 \mathrm{E}-02$ & $9.0101 \mathrm{E}-02$ \\
\hline $\mathrm{f} 2-57$ & Zakharov & 2 & \begin{tabular}{|l|}
$1.4286 \mathrm{E}-07$ \\
\end{tabular} & 6.8890E-05 & $6.9831 \mathrm{E}-05$ & \begin{tabular}{|l|}
$6.0584 \mathrm{E}-07$ \\
\end{tabular} & 6.6225E-06 & 6.2012E-06 \\
\hline $\mathrm{f} 2-58$ & Zettl & 2 & 2.6937E- 08 & $8.7643 \mathrm{E}-06$ & $1.0503 \mathrm{E}-05$ & \begin{tabular}{|l|}
$9.2581 \mathrm{E}-09$ \\
\end{tabular} & \begin{tabular}{|l}
$1.9735 \mathrm{E}-06$ \\
\end{tabular} & 4.2604E-06 \\
\hline f2-59 & 3-Hump Camel-Back & 2 & 5.7882E- 08 & 2.8687E-05 & 5.8124E-05 & \begin{tabular}{|l}
$9.8715 \mathrm{E}-08$ \\
\end{tabular} & \begin{tabular}{|l}
$5.3205 \mathrm{E}-06$ \\
\end{tabular} & $7.9850 \mathrm{E}-06$ \\
\hline$f 2-60$ & 6-Hump Camel-Back & 2 & $2.3455 \mathrm{E}-07$ & 9.1217E-05 & $1.2481 \mathrm{E}-04$ & $3.4081 \mathrm{E}-08$ & $1.5711 \mathrm{E}-05$ & $2.2781 E-05$ \\
\hline
\end{tabular}


Table 2b. Comparison of results over 30 trails of the original and modified versions of PMB-BBO, where "Best" means the best error, "Mean" indicates the mean best error, and "StdDev" stands for the standard deviation - (where $n \neq 2$ )

\begin{tabular}{|c|c|c|c|c|c|c|c|c|}
\hline \multirow{3}{*}{ Func. \# } & \multirow{3}{*}{ Function Name } & \multirow{3}{*}{$\mathbf{n}$} & \multicolumn{6}{|c|}{ Biogeography Based Optimization (BBO) } \\
\hline & & & \multicolumn{3}{|c|}{ Original Partial Migration Based } & \multicolumn{3}{|c|}{ Modified Partial Migration Based } \\
\hline & & & Best & Mean & StdDev & Best & Mean & StdDev \\
\hline f1-01 & Mineshaft F1 & 1 & $6.2998 \mathrm{E}-01$ & 7.5377E-01 & $7.4896 \mathrm{E}-02$ & 4.4885E-01 & \begin{tabular}{|l|}
$7.4142 \mathrm{E}-01$ \\
\end{tabular} & $1.0203 \mathrm{E}-01$ \\
\hline f1-02 & Mineshaft F2 & 1 & \begin{tabular}{|l|}
$2.8719 E-09$ \\
\end{tabular} & $4.6768 \mathrm{E}-04$ & 6.6885E-04 & 3.9974E-08 & 3.9916E-04 & $8.2865 \mathrm{E}-04$ \\
\hline f1-03 & Shekel's F1 & 1 & $9.8030 \mathrm{E}-08$ & 2.8723E-05 & $4.2939 \mathrm{E}-05$ & 6.7958E-09 & $1.2670 \mathrm{E}-04$ & 4.0902E-04 \\
\hline f1-04 & Shekel's F2 & 1 & $8.1958 \mathrm{E}-10$ & $2.1546 \mathrm{E}-05$ & $5.2185 \mathrm{E}-05$ & $8.7502 \mathrm{E}-09$ & $5.4862 \mathrm{E}-05$ & $8.6120 \mathrm{E}-05$ \\
\hline f1-05 & Shekel's F3 & 1 & $1.7454 \mathrm{E}-08$ & $7.4624 \mathrm{E}-05$ & 1.8726E-04 & $1.4736 \mathrm{E}-10$ & 5.1717E-05 & $8.7380 \mathrm{E}-05$ \\
\hline f1-06 & Stron.-Zilin.-Shalt. & 1 & 5.7915E-11 & $1.0865 \mathrm{E}-07$ & 2.4327E-07 & $1.0768 \mathrm{E}-10$ & $2.5748 \mathrm{E}-07$ & $6.8094 \mathrm{E}-07$ \\
\hline f1-07 & Suharev & 1 & $1.5944 \mathrm{E}-11$ & 9.3043E- 08 & $1.2955 \mathrm{E}-07$ & $1.1613 \mathrm{E}-11$ & $1.8424 \mathrm{E}-07$ & 4.0888E-07 \\
\hline f1-08 & Zilinskas F2 & 1 & $1.4002 \mathrm{E}-09$ & $2.2730 \mathrm{E}-06$ & 5.2141E-06 & $1.0064 \mathrm{E}-09$ & 9.9106E-06 & $2.2533 \mathrm{E}-05$ \\
\hline f3-01 & Box-Betts & 3 & \begin{tabular}{|l|}
$2.3366 \mathrm{E}-08$ \\
\end{tabular} & $1.4450 \mathrm{E}-06$ & \begin{tabular}{|l|}
$1.1712 \mathrm{E}-06$ \\
\end{tabular} & 2.3614E-09 & \begin{tabular}{|l|}
$1.0091 E-06$ \\
\end{tabular} & $1.2014 \mathrm{E}-06$ \\
\hline f3-02 & Hartman's F1 & 3 & 7.6177E-06 & $5.0374 \mathrm{E}-04$ & $3.8211 \mathrm{E}-04$ & $1.0262 \mathrm{E}-06$ & 3.8549E-05 & $7.4211 \mathrm{E}-05$ \\
\hline f3-03 & Helical Valley & 3 & $8.1299 \mathrm{E}-02$ & $8.4147 \mathrm{E}-01$ & $6.2500 \mathrm{E}-01$ & 2.3847E-03 & $5.2274 \mathrm{E}-01$ & $6.8708 \mathrm{E}-01$ \\
\hline f3-04 & Levy F8 & 3 & 6.4096E-06 & $1.2455 \mathrm{E}-03$ & $1.2789 \mathrm{E}-03$ & 4.8947E-07 & 5.2271E-05 & $6.1623 \mathrm{E}-05$ \\
\hline f3-05 & Meyer and Roth & 3 & 9.1336E-06 & $1.0188 \mathrm{E}-04$ & $8.3202 E-05$ & $4.5474 \mathrm{E}-06$ & $7.0623 \mathrm{E}-05$ & $9.3791 \mathrm{E}-05$ \\
\hline f3-06 & Perm No.1 & 3 & $1.6149 \mathrm{E}-01$ & $1.2030 \mathrm{E}+00$ & 7.6733E-01 & $4.0283 \mathrm{E}-03$ & $5.7516 \mathrm{E}-01$ & 4.9994E-01 \\
\hline f4-01 & Corana (or Ingber) & 4 & $3.5444 \mathrm{E}+00$ & $8.6925 \mathrm{E}+01$ & $6.1928 \mathrm{E}+01$ & $0.0000 E+00$ & $1.1788 \mathrm{E}+01$ & $1.2559 E+01$ \\
\hline $\mathrm{f4-02}$ & Kowalik & 4 & $3.9927 \mathrm{E}-04$ & $8.0573 \mathrm{E}-04$ & $3.2529 \mathrm{E}-04$ & $2.5735 \mathrm{E}-04$ & $6.2274 \mathrm{E}-04$ & $3.0950 \mathrm{E}-04$ \\
\hline f4-03 & Miele and Cantrell & 4 & $8.0518 \mathrm{E}-09$ & $1.2520 \mathrm{E}-06$ & $1.2340 \mathrm{E}-06$ & $1.2172 \mathrm{E}-10$ & $1.4661 \mathrm{E}-06$ & $3.9810 \mathrm{E}-06$ \\
\hline $\mathrm{f} 4-04$ & Powell's Quartic & 4 & $2.8518 \mathrm{E}-02$ & $1.8301 \mathrm{E}+00$ & $2.0186 \mathrm{E}+00$ & $5.9062 \mathrm{E}-03$ & 2.6144E-01 & $3.1940 \mathrm{E}-01$ \\
\hline f4-05 & Neumaier F2 & 4 & 9.7017E-03 & $3.9273 \mathrm{E}-02$ & $2.4992 \mathrm{E}-02$ & $6.8860 \mathrm{E}-03$ & $2.8289 \mathrm{E}-02$ & $2.3559 \mathrm{E}-02$ \\
\hline f4-06 & Wood (or Colville) & 4 & $1.5438 \mathrm{E}+00$ & $7.1389 \mathrm{E}+00$ & $3.4273 \mathrm{E}+00$ & 1.2867E-01 & $1.9675 \mathrm{E}+00$ & $1.3293 \mathrm{E}+00$ \\
\hline $\mathrm{f} 5-01$ & AMGM & 5 & $9.8524 \mathrm{E}-10$ & $1.4399 \mathrm{E}-07$ & $2.2968 \mathrm{E}-07$ & 3.1111E-11 & 4.1812E-09 & $8.7922 \mathrm{E}-09$ \\
\hline $\mathrm{f5}-02$ & borne No.1 & 5 & \begin{tabular}{|l}
$1.1839 \mathrm{E}-02$ \\
\end{tabular} & $1.1988 \mathrm{E}-01$ & $9.1466 \mathrm{E}-02$ & $1.1325 \mathrm{E}-02$ & $1.3801 \mathrm{E}-01$ & $1.0496 \mathrm{E}-01$ \\
\hline f5-03 & SODP & 5 & 7.2433E-07 & 6.9565E-05 & $8.3596 \mathrm{E}-05$ & 6.3699E-09 & $1.6674 \mathrm{E}-05$ & $2.4309 \mathrm{E}-05$ \\
\hline f5-04 & Styblinski-Tang & 5 & $8.1139 \mathrm{E}-02$ & $8.6368 \mathrm{E}-01$ & $8.0724 \mathrm{E}-01$ & 3.4485E-03 & $6.1622 \mathrm{E}-02$ & $5.0114 \mathrm{E}-02$ \\
\hline f6-01 & Hartman's F2 & 6 & $1.8781 \mathrm{E}-03$ & $6.9158 \mathrm{E}-02$ & 6.0706E-02 & 7.1304E-04 & $3.8071 \mathrm{E}-02$ & $5.4616 \mathrm{E}-02$ \\
\hline$f 6-02$ & Perm No.2 & 6 & $2.9814 \mathrm{E}-01$ & $1.0219 \mathrm{E}+00$ & 6.1973E-01 & $1.8974 \mathrm{E}-02$ & $5.7573 \mathrm{E}-01$ & $6.0020 \mathrm{E}-01$ \\
\hline f9-01 & ANNs XOR & 9 & 7.5664E-04 & $5.7103 \mathrm{E}-03$ & $3.6158 \mathrm{E}-03$ & $7.9878 \mathrm{E}-04$ & $6.8751 \mathrm{E}-03$ & $2.2983 \mathrm{E}-03$ \\
\hline$f 9-02$ & Price's Transistor & 9 & $2.5525 \mathrm{E}+01$ & $1.3334 \mathrm{E}+02$ & $5.4565 \mathrm{E}+01$ & $9.1948 \mathrm{E}+00$ & $9.0453 E+01$ & $4.1274 E+01$ \\
\hline f9-03 & torn's Chebyshev & 9 & $9.7939 \mathrm{E}+03$ & $5.4462 \mathrm{E}+04$ & $3.6762 \mathrm{E}+04$ & $3.5246 \mathrm{E}+03$ & $2.6181 E+04$ & $2.2022 E+04$ \\
\hline$f 10-01$ & Epistatic Michalewicz & 10 & $4.2122 \mathrm{E}-01$ & $1.2966 \mathrm{E}+00$ & 6.2987E-01 & $1.5307 \mathrm{E}-01$ & 6.7944E-01 & $3.4882 \mathrm{E}-01$ \\
\hline $\mathrm{f} 10-02$ & Katsuura & 10 & $2.1784 \mathrm{E}-01$ & 5.0836E-01 & $1.4062 \mathrm{E}-01$ & $8.0454 \mathrm{E}-02$ & $2.9182 \mathrm{E}-01$ & \begin{tabular}{|l}
$1.1279 \mathrm{E}-01$ \\
\end{tabular} \\
\hline f10-03 & Odd Square & 10 & $9.0095 \mathrm{E}-01$ & $1.0167 E+00$ & 4.3942E-02 & $9.6634 \mathrm{E}-01$ & $1.0366 \mathrm{E}+00$ & 3.0877E-02 \\
\hline $\mathrm{f} 10-04$ & Paviani & 10 & $6.2946 \mathrm{E}-03$ & $2.0765 \mathrm{E}-02$ & $1.3830 \mathrm{E}-02$ & $1.3197 \mathrm{E}-03$ & 5.5884E-03 & $4.0332 \mathrm{E}-03$ \\
\hline$f 15-01$ & Dixon-Price & 15 & $5.6066 \mathrm{E}-01$ & $2.1042 \mathrm{E}+00$ & $1.3961 \mathrm{E}+00$ & 7.3983E-01 & $1.4613 \mathrm{E}+00$ & 5.4173E-01 \\
\hline f15-02 & Neumaier F3 (or Trid) & 15 & $1.0026 \mathrm{E}+02$ & $9.5503 E+02$ & $6.7827 \mathrm{E}+02$ & $8.9477 \mathrm{E}+01$ & $8.7253 \mathrm{E}+02$ & 7.7831E+02 \\
\hline f15-03 & Normalized Rana & 15 & $2.9324 E+01$ & $5.6653 \mathrm{E}+01$ & $1.2546 \mathrm{E}+01$ & $2.9923 \mathrm{E}+01$ & $4.6883 \mathrm{E}+01$ & $9.8307 E+00$ \\
\hline$f 17-01$ & Bent Cigar & 17 & $9.2454 \mathrm{E}+05$ & $2.6185 \mathrm{E}+06$ & $1.2377 \mathrm{E}+06$ & $5.3826 \mathrm{E}+05$ & $1.3379 \mathrm{E}+06$ & $5.7265 E+05$ \\
\hline$f 17-02$ & Defl. Corrug. Spring & 17 & 6.2664E-01 & $1.3368 \mathrm{E}+00$ & 5.0800E-01 & $6.2664 \mathrm{E}-01$ & 9.9217E-01 & $3.9077 \mathrm{E}-01$ \\
\hline f17-03 & Infinity (or Csendes) & 17 & $4.6802 \mathrm{E}-14$ & $1.6281 \mathrm{E}-11$ & $2.4605 \mathrm{E}-11$ & 4.1422E-15 & $2.4643 \mathrm{E}-12$ & $4.6031 \mathrm{E}-12$ \\
\hline $\mathrm{f} 20-01$ & Alpine & 20 & $1.7629 \mathrm{E}-02$ & $3.6322 \mathrm{E}-02$ & $1.2919 \mathrm{E}-02$ & $1.5741 \mathrm{E}-02$ & 2.8971E-02 & $1.0286 \mathrm{E}-02$ \\
\hline$f 20-02$ & Quintic & 20 & $2.0141 E+00$ & $3.2993 \mathrm{E}+00$ & 7.3579E-01 & $1.4342 \mathrm{E}+00$ & $2.6384 E+00$ & $5.6576 \mathrm{E}-01$ \\
\hline $\mathrm{f} 20-03$ & Pathological & 20 & $1.7044 \mathrm{E}+00$ & $2.6537 E+00$ & 4.2252E-01 & $2.1295 \mathrm{E}+00$ & $2.5968 \mathrm{E}+00$ & $3.8864 \mathrm{E}-01$ \\
\hline $\mathrm{f} 30-01$ & Ackley & 30 & $6.3026 \mathrm{E}-01$ & $9.9236 \mathrm{E}-01$ & $2.3065 \mathrm{E}-01$ & 5.6622E-01 & 9.3734E-01 & 2.1466E-01 \\
\hline $\mathrm{f} 30-02$ & Gen. Griewank & 30 & $8.6708 \mathrm{E}-01$ & $1.0263 \mathrm{E}+00$ & $3.4402 \mathrm{E}-02$ & $8.4581 \mathrm{E}-01$ & $1.0131 \mathrm{E}+00$ & $4.7757 \mathrm{E}-02$ \\
\hline f30-03 & Gen. Penalized F1 & 30 & 5.0934E-03 & $2.9591 \mathrm{E}-02$ & 3.2559E-02 & $1.6288 \mathrm{E}-03$ & $1.7078 \mathrm{E}-02$ & $1.6484 \mathrm{E}-02$ \\
\hline f30-04 & Gen. Penalized F2 & 30 & $9.3001 \mathrm{E}-02$ & $1.6875 \mathrm{E}-01$ & 6.0757E-02 & $8.2945 \mathrm{E}-02$ & 1.8237E-01 & $7.5631 \mathrm{E}-02$ \\
\hline f30-05 & Gen. Rastrigin & 30 & 9.4594E-01 & $1.9351 \mathrm{E}+00$ & $6.4580 \mathrm{E}-01$ & 7.5521E-01 & $1.7540 \mathrm{E}+00$ & 7.1453E-01 \\
\hline f30-06 & Gen. Rosenbrock & 30 & $1.0517 \mathrm{E}+02$ & $2.6058 \mathrm{E}+02$ & $7.9555 E+01$ & $8.5320 \mathrm{E}+01$ & $2.4504 \mathrm{E}+02$ & $9.3473 \mathrm{E}+01$ \\
\hline f30-07 & Gen. Schwefel F2.26 & 30 & \begin{tabular}{|l}
$4.4291 E-06$ \\
\end{tabular} & $1.3876 \mathrm{E}-05$ & 5.7466E-06 & $4.6460 \mathrm{E}-06$ & $1.0766 \mathrm{E}-05$ & 3.8181E-06 \\
\hline f30-08 & Mishra F1 & 30 & $1.3229 \mathrm{E}-01$ & $1.8511 \mathrm{E}-01$ & $3.4109 \mathrm{E}-02$ & $1.1668 \mathrm{E}-01$ & $1.6764 \mathrm{E}-01$ & $2.7709 \mathrm{E}-02$ \\
\hline f30-09 & Mishra F2 & 30 & $1.2195 \mathrm{E}-01$ & $1.9327 \mathrm{E}-01$ & $3.4082 \mathrm{E}-02$ & 1.1973E-01 & $1.6742 \mathrm{E}-01$ & $3.0336 \mathrm{E}-02$ \\
\hline f30-10 & Quartic & 30 & $2.8562 \mathrm{E}-07$ & $1.2406 \mathrm{E}-06$ & $1.2872 \mathrm{E}-06$ & 4.9473E- 08 & 9.6111E-07 & $1.1137 \mathrm{E}-06$ \\
\hline f30-11 & Schwefel F1.2 & 30 & $4.2433 \mathrm{E}+02$ & $1.9527 \mathrm{E}+04$ & $1.1268 \mathrm{E}+04$ & $1.0214 \mathrm{E}+00$ & $2.6767 \mathrm{E}+02$ & $2.9316 \mathrm{E}+02$ \\
\hline f30-12 & Schwefel F2.21 & 30 & $3.2007 E+00$ & $6.2387 \mathrm{E}+00$ & 1.1469E+00 & $4.3096 \mathrm{E}+00$ & $5.5198 \mathrm{E}+00$ & $9.0466 \mathrm{E}-01$ \\
\hline f30-13 & Schwefel F2.22 & 30 & 4.0024E-01 & 7.0184E-01 & $1.3458 \mathrm{E}-01$ & 4.5480E-01 & 7.0286E-01 & 1.3019E-01 \\
\hline f30-14 & Sphere & 30 & $1.8518 \mathrm{E}+00$ & $3.8843 \mathrm{E}+00$ & $1.3964 \mathrm{E}+00$ & $1.5508 \mathrm{E}+00$ & $3.3657 \mathrm{E}+00$ & $1.2515 \mathrm{E}+00$ \\
\hline f30-15 & Step & 30 & $2.0000 \mathrm{E}+00$ & $4.7333 \mathrm{E}+00$ & $1.8245 E+00$ & $0.0000 E+00$ & $4.1000 \mathrm{E}+00$ & $1.8859 \mathrm{E}+00$ \\
\hline$f 60-01$ & Hyper-Ellipsoid & 60 & 5.2404E-01 & $1.0131 \mathrm{E}+00$ & 3.8482E-01 & 5.1024E-01 & $9.0328 \mathrm{E}-01$ & 2.6241E-01 \\
\hline$f 60-02$ & Qing & 60 & $3.5937 \mathrm{E}+03$ & $5.9785 E+03$ & $1.6586 \mathrm{E}+03$ & $3.5094 \mathrm{E}+03$ & $6.4488 \mathrm{E}+03$ & $2.0255 \mathrm{E}+03$ \\
\hline $660-03$ & Salomon & 60 & $2.2999 \mathrm{E}+00$ & $3.0172 \mathrm{E}+00$ & 3.5403E-01 & $2.2999 \mathrm{E}+00$ & $2.7949 E+00$ & $2.5704 \mathrm{E}-01$ \\
\hline
\end{tabular}


Although, the modified version of PMB-BBO shows enhanced results, for some very hard test functions, like Price's Transistor, Storn's Chebyshev, Trid "or Neumaier F3", Normalized Rana, Bent Cigar, Qing, Generalized Rosenbrock and Schwefel F1.2 problems, both versions failed to converge to the optimal solutions. However, as a comparison, the proposed version outperform the original version on most of these test functions.

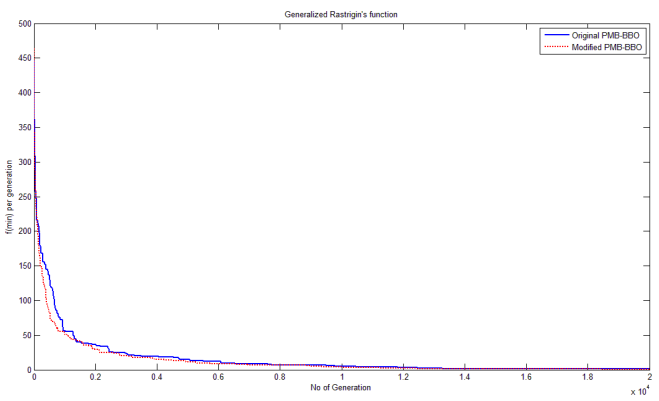

(a)

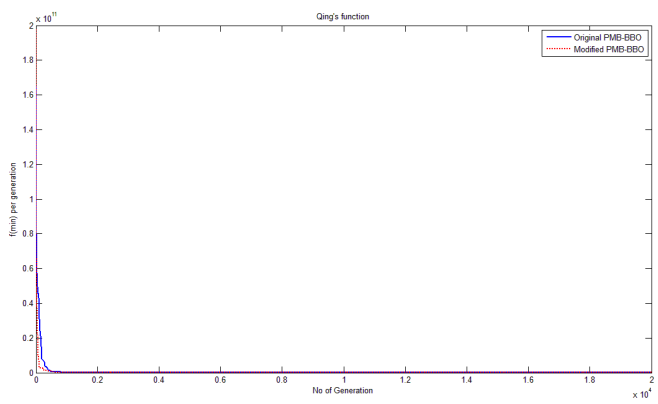

(c)

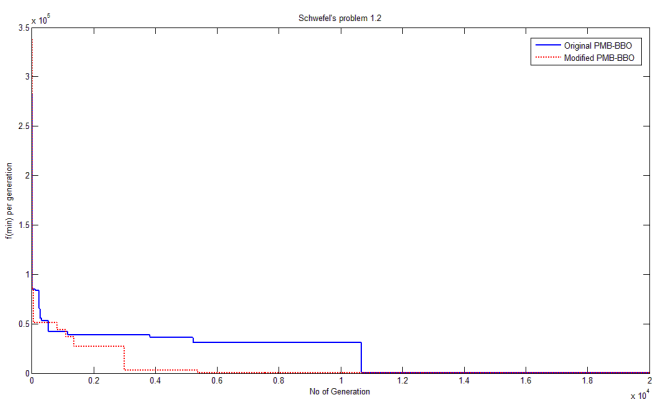

(b)

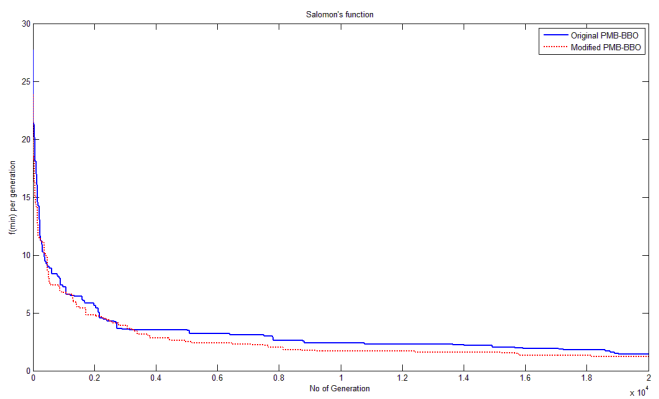

(d)

Figure 5. Curves of fitness functions of the original and modified PMB for some selected functions.(a) f3005, (b) f30-11, (c) f60-02, (d) f60-03

Table 3 shows the CPU time comparison between the modified PMB-BBO and the original PMBBBO. It can be clearly seen from Table 3 that the modified version can save around $32.32 \%$ of the CPU time, which means that it is faster than even the simplified partial migration model (SPMB-BBO) by around $24.76 \%$ [20].

Table 3. Normalized CPU times on 60-dimensional test functions

\begin{tabular}{|lccc|}
\hline \multirow{2}{*}{ Function } & \multicolumn{2}{c}{ PMB-BBO Versions } & CPU Time Saving \\
\cline { 2 - 3 } & Original ver. & Modified ver. & (\%) \\
\hline Hyper-Ellipsoid & $1.4617 \mathrm{E}+00$ & $\mathbf{1 . 0 0 0 0 \mathrm { E } + 0 0}$ & 31.58437697 \\
Qing & $1.4605 \mathrm{E}+00$ & $\mathbf{1 . 0 0 0 0 \mathrm { E } + 0 0}$ & 31.5305639 \\
Salomon & $1.5104 \mathrm{E}+00$ & $\mathbf{1 . 0 0 0 0 \mathrm { E } + 0 0}$ & 33.79377207 \\
\hline Avg. CPU Time & $1.4775 \mathrm{E}+00$ & $\mathbf{1 . 0 0 0 0 \mathrm { E } + 0 0}$ & 32.3195104 \\
\hline
\end{tabular}

\subsection{Discussions}

As a comparison between the four original models of BBO (PMB, SMB, SPMB and SSMB), PMB-BBO gives the best performance when the given problem is hard, has large upper and lower limits of search spaces, high-dimensional and/or the number of islands or population size is small [20]. However, PMB-BBO lacks the exploration [18]. Therefore, in this study, the root problem 
that causes the poor exploration is solved by using an integer random function which provides a pseudorandom integers from a uniform discrete distribution. Furthermore, the exploitation is improved by keeping the non-mutated solutions away from any corruption by clear duplication process.

\section{Conclusions ANd Suggestions}

This study proposed some modifications to improve the performance of the original form of PMB-BBO without using any complicated models for immigration and emigration rates. It is shown that the simplified linear model still can give good results if the root problems of the migration and mutation stages are solved. An extensive testing of the original and the proposed modified versions of PMB-BBO through 120 test functions shows that the performance of the modified version of PMB-BBO is better on Best, Mean, and StdDev than that of the original version.

The proposed modification can be used as a basis for modifying the existing modified BBOs in other literatures. For example, the blended-BBO which is presented in [14] can be a great addonto this modified PMB-BBO. Furthermore, even if it is required to employ the complicated models of immigration and emigration rates, such as the generalized sinusoidal migration modelin [16].The generalized sinusoidal migration model is based on an old study that was done by James A. MacMahon in 1987 [19], and was mentioned in ch.3 of [4]. This complicated model shows great performance, and it is very interesting to re-test this model with considering the proposed essential modifications that are described in this study.

\section{APPENDIX}

This appendix contains a complete list of all the involved test functions that are collected from various sources where some of them are hard to be found while the other, especially the old functions, are corrected analytically before being used here. This is because they are available in their original sources with approximated global solutions. Perhaps due limited computing capability in that time. These 120 test functions are spread through references [22-64], and some of these references have a collection of test functions, which means that the popular test functions can be found in different locations, while the others are located in one or few locations. In addition, sometimes the information about test functions are available in different references. For more information regarding any test function, please refer to the related reference(s).

\begin{tabular}{|c|c|c|c|c|c|}
\hline$f \#$ & Function Name & $\begin{array}{c}\text { Dimension } \\
(\mathbf{n})\end{array}$ & Variables Bounds & Global Optimum & References \\
\hline$f 1-01$ & Mineshaft F1 & 1 & $0 \leq x \leq 10$ & 1.380487165157852 & [63] \\
\hline$f 1-02$ & Mineshaft F2 & 1 & $-10 \leq x \leq 10$ & -1.416353520337699 & [63] \\
\hline$f 1-03$ & Shekel's F1 & 1 & $0 \leq x \leq 10$ & $\begin{array}{l}-10.1531987550848817763568 \\
39400251\end{array}$ & {$[23,24]$} \\
\hline$f 1-04$ & Shekel's F2 & 1 & $0 \leq x \leq 10$ & $\begin{array}{l}-10.4028220447077753290705 \\
18200751\end{array}$ & {$[23,24]$} \\
\hline$f 1-05$ & Shekel's F3 & 1 & $0 \leq x \leq 10$ & $\begin{array}{l}-10.5362902992947171054273 \\
57601002\end{array}$ & {$[23,24]$} \\
\hline$f 1-06$ & $\begin{array}{l}\text { Strongin-Zilinskas- } \\
\text { Shaltyanis }\end{array}$ & 1 & $3 \leq x \leq 7.5$ & -1.601307546494396 & [25] \\
\hline$f 1-07$ & Suharev & 1 & $0 \leq x \leq 1$ & -1 & {$[25]$} \\
\hline$f 1-08$ & ZilinskasF2 & 1 & $0 \leq x \leq 1$ & -1.125 & {$[25]$} \\
\hline$f 2-01$ & Aluffi-Pentini & 2 & $-10 \leq x_{i} \leq 10$ & -0.352386073800034 & {$[26]$} \\
\hline$f 2-02$ & Banana Shape & 2 & $\begin{array}{l}-1.5 \leq x_{1} \leq 1.5 \\
-2.5 \leq x_{2} \leq 0.5\end{array}$ & -25 & [27] \\
\hline$f 2-03$ & Beale & 2 & $-4.5 \leq x_{i} \leq 4.5$ & 0 & {$[28,50]$} \\
\hline
\end{tabular}




\begin{tabular}{|c|c|c|c|c|c|}
\hline$f 2-04$ & Becker-Lago & 2 & $-10 \leq x_{i} \leq 10$ & 0 & [26] \\
\hline$f 2-05$ & Bird & 2 & $-2 \pi \leq x_{i} \leq 2 \pi$ & -106.7645367198034 & {$[29,33,34]$} \\
\hline$f 2-06$ & BohachevskyF1 & 2 & $-50 \leq x_{i} \leq 50$ & 0 & {$[26,30]$} \\
\hline$f 2-07$ & BohachevskyF2 & 2 & $-50 \leq x_{i} \leq 50$ & 0 & {$[26,30]$} \\
\hline$f 2-08$ & BohachevskyF3 & 2 & $-50 \leq x_{i} \leq 50$ & 0 & {$[30]$} \\
\hline$f 2-09$ & Booth & 2 & $-10 \leq x_{i} \leq 10$ & 0 & {$[28,31]$} \\
\hline$f 2-10$ & Branin RCOS & 2 & $\begin{array}{c}-5 \leq x_{1} \leq 10 \\
0 \leq x_{2} \leq 15\end{array}$ & 0.39788735772973816 & {$[31,32]$} \\
\hline$f 2-11$ & BukinF4 & 2 & $-15 \leq x_{i} \leq 5$ & 0 & {$[33]$} \\
\hline$f 2-12$ & BukinF6 & 2 & $-15 \leq x_{i} \leq 5$ & 0 & [33] \\
\hline$f 2-13$ & Carrom Table & 2 & $-10 \leq x_{i} \leq 10$ & -24.15681551650653 & {$[34]$} \\
\hline$f 2-14$ & Chichinadze & 2 & $-30 \leq x_{i} \leq 30$ & -42.94438701899098 & {$[34]$} \\
\hline$f 2-15$ & Complex & 2 & $-2 \leq x_{i} \leq 2$ & 0 & {$[35]$} \\
\hline$f 2-16$ & Cosine Mixture & 2 & $-1 \leq x_{i} \leq 1$ & 0.2 & {$[38]$} \\
\hline$f 2-17$ & Cross In Tray & 2 & $-15 \leq x_{i} \leq 15$ & -2.062611870822739 & {$[33,34]$} \\
\hline$f 2-18$ & Cross Leg Table & 2 & $-10 \leq x_{i} \leq 10$ & -1 & {$[33,34]$} \\
\hline$f 2-19$ & Crowned Cross & 2 & $-10 \leq x_{i} \leq 10$ & 0.0001 & {$[33,34]$} \\
\hline$f 2-20$ & Davis & 2 & $-100 \leq x_{i} \leq 100$ & 0 & {$[35]$} \\
\hline$f 2-21$ & Decanomial & 2 & $-10 \leq x_{i} \leq 10$ & 0 & {$[34]$} \\
\hline$f 2-22$ & Dekkers-Aarts & 2 & $-20 \leq x_{i} \leq 20$ & -24777 & [26] \\
\hline$f 2-23$ & Drop Wave & 2 & $-5.12 \leq x_{i} \leq 5.12$ & -1 & {$[34,36]$} \\
\hline$f 2-24$ & Easom & 2 & $-10 \leq x_{i} \leq 10$ & -1 & {$[26,31,32,36,37]$} \\
\hline$f 2-25$ & Egg Holder & 2 & $-512 \leq x_{i} \leq 512$ & $-959.640662711($ for $n=2)$ & {$[31,33,34]$} \\
\hline$f 2-26$ & EXP2 & 2 & $0 \leq x_{i} \leq 20$ & 0 & {$[31,34]$} \\
\hline$f 2-27$ & Freudenstein-Roth & 2 & $-10 \leq x_{i} \leq 10$ & 0 & {$[39,50]$} \\
\hline$f 2-28$ & Giunta & 2 & $-1 \leq x_{i} \leq 1$ & $\begin{array}{c}0.06447042053690566 \\
(\text { for } n=2)\end{array}$ & {$[33,34]$} \\
\hline$f 2-29$ & Goldstein-Price & 2 & $-2 \leq x_{i} \leq 2$ & 3 & $\begin{array}{l}{[26,31,32,34,35,} \\
36,40,41]\end{array}$ \\
\hline$f 2-30$ & Himmelblau & 2 & $-6 \leq x_{i} \leq 6$ & 0 & $\begin{array}{l}{[25,31,34,35,41,} \\
43]\end{array}$ \\
\hline$f 2-31$ & Holder Table & 2 & $-10 \leq x_{i} \leq 10$ & -19.20850256788675 & {$[33,34]$} \\
\hline$f 2-32$ & Hosaki & 2 & $0 \leq x_{i} \leq 10$ & -2.345811576101292 & {$[26,31,34]$} \\
\hline$f 2-33$ & Kearfott & 2 & $-3 \leq x_{i} \leq 4$ & 0 & {$[43,44,45]$} \\
\hline$f 2-34$ & Inverted Cosine Wave & 2 & $-5 \leq x_{i} \leq 5$ & $-n+1$ & [37] \\
\hline$f 2-35$ & $\begin{array}{c}\text { Levy F3 (Shubert or } \\
\text { Hansen) }\end{array}$ & 2 & $-10 \leq x_{i} \leq 10$ & -176.5417931365915 & {$[26,31,42]$} \\
\hline$f 2-36$ & Levy F5 & 2 & $-10 \leq x_{i} \leq 10$ & -176.1375 & {$[31,46]$} \\
\hline$f 2-37$ & Matyas & 2 & $-10 \leq x_{i} \leq 10$ & 0 & {$[28,31,34,37]$} \\
\hline$f 2-38$ & McCormick & 2 & $\begin{array}{c}-1.5 \leq x_{1} \leq 4 \\
-3 \leq x_{2} \leq 4\end{array}$ & -1.913222954981037 & {$[26,31,33,34]$} \\
\hline$f 2-39$ & Michalewicz & 2 & $0 \leq x_{i} \leq \pi$ & $\begin{array}{c}-1.801303228593281 \\
(\text { for } n=2)\end{array}$ & {$[36,37]$} \\
\hline$f 2-40$ & Muller-Brown Surface & 2 & $\begin{array}{l}-1.5 \leq x_{1} \leq 1 \\
-0.5 \leq x_{2} \leq 2.5\end{array}$ & -146.6995172099539 & {$[47,48]$} \\
\hline$f 2-41$ & Parsopoulos & 2 & $-5 \leq x_{i} \leq 5$ & 0 & [41] \\
\hline$f 2-42$ & Peaks & 2 & $-4 \leq x_{i} \leq 4$ & -6.551133332622496 & [49] \\
\hline$f 2-43$ & Pen Holder & 2 & $-11 \leq x_{i} \leq 11$ & -0.9635348327265058 & {$[33,34]$} \\
\hline$f 2-44$ & Powell's Badly Scaled & 2 & $-10 \leq x_{i} \leq 10$ & 0 & {$[50,51,52]$} \\
\hline$f 2-45$ & Sawtoothxy & 2 & $-20 \leq x_{i} \leq 20$ & 0 & {$[49,54]$} \\
\hline$f 2-46$ & Schaffer's F1 & 2 & $-100 \leq x_{i} \leq 100$ & 0 & {$[26,31,33,48]$} \\
\hline$f 2-47$ & Schaffer's F2 & 2 & $-100 \leq x_{i} \leq 100$ & 0 & {$[26,31,33,48]$} \\
\hline$f 2-48$ & Shekel's Foxholes & 2 & $-65.536 \leq x_{i} \leq 65.536$ & $\begin{array}{l}0.998003837794449325873406 \\
851315\end{array}$ & {$[23,36,54]$} \\
\hline$f 2-49$ & Sinusoidal Problem & 2 & $0 \leq x_{i} \leq 180^{\circ}$ & -3.5 & {$[26]$} \\
\hline$f 2-50$ & Stenger & 2 & $-1 \leq x_{i} \leq 4$ & 0 & [35] \\
\hline$f 2-51$ & Storn & 2 & $-4 \leq x_{i} \leq 4$ & -18.0587 & {$[41]$} \\
\hline$f 2-52$ & Stretched V & 2 & $-10 \leq x_{i} \leq 10$ & $0($ for $n=2)$ & {$[31,34]$} \\
\hline$f 2-53$ & Test Tube Holder & 2 & $-10 \leq x_{i} \leq 10$ & -10.872299901558 & {$[33,34]$} \\
\hline
\end{tabular}


Computer Science \& Information Technology (CS \& IT)

\begin{tabular}{|c|c|c|c|c|c|}
\hline$f 2-54$ & Treccani & 2 & $-5 \leq x_{i} \leq 5$ & 0 & {$[34,55]$} \\
\hline$f 2-55$ & TrefethenF4 & 2 & $\begin{array}{l}-6.5 \leq x_{1} \leq 6.5 \\
-4.5 \leq x_{2} \leq 4.5\end{array}$ & -3.3068686474 & {$[31,34,54]$} \\
\hline f2-56 & Tripod & 2 & $-100 \leq x_{i} \leq 100$ & 0 & [37] \\
\hline$f 2-57$ & Zakharov & 2 & $-5 \leq x_{i} \leq 10$ & 0 & {$[32,37]$} \\
\hline$f 2-58$ & Zettl & 2 & $-1 \leq x_{i} \leq 5$ & -0.003791237220468656 & {$[31,33,34]$} \\
\hline$f 2-59$ & 3-Hump Camel-Back & 2 & $-5 \leq x_{i} \leq 5$ & 0 & {$[26,31,33,40]$} \\
\hline$f 2-60$ & 6-Hump Camel-Back & 2 & $-5 \leq x_{i} \leq 5$ & -1.031628453489877 & $\begin{array}{l}{[23,26,31,34,36,} \\
37,40]\end{array}$ \\
\hline f3-01 & Box-Betts & 3 & $\begin{array}{c}0.9 \leq x_{1}, x_{3} \leq 1.2 \\
9 \leq x_{2} \leq 11.2\end{array}$ & 0 & {$[31,34]$} \\
\hline f3-02 & Hartman's F1 & 3 & $0 \leq x_{i} \leq 1$ & -3.86278214782076 & $\begin{array}{l}{[23,26,30,31,32,} \\
34,37,40]\end{array}$ \\
\hline f3-03 & Helical Valley & 3 & $-100 \leq x_{i} \leq 100$ & 0 & $\begin{array}{l}{[26,34,39,50,52,} \\
56]\end{array}$ \\
\hline f3-04 & Levy F8 & 3 & $-10 \leq x_{i} \leq 10$ & 0 & {$[31,41]$} \\
\hline f3-05 & Meyer and Roth & 3 & $-20 \leq x_{i} \leq 20$ & $0.4 \times 10^{-4}$ & {$[26]$} \\
\hline f3-06 & Perm F1 & 3 & $-n \leq x_{i} \leq n+1$ & 0 & {$[34,55]$} \\
\hline$f 4-01$ & Corana (or Ingber) & 4 & $-100 \leq x_{i} \leq 100$ & 0 & {$[31,34,57]$} \\
\hline$f 4-02$ & Kowalik & 4 & $-5 \leq x_{i} \leq 5$ & $\begin{array}{l}0.000307485987805604216840 \\
4344971009\end{array}$ & $\begin{array}{l}{[23,26,30,31,34,} \\
37]\end{array}$ \\
\hline f4-03 & Miele and Cantrell & 4 & $-1 \leq x_{i} \leq 1$ & 0 & {$[26]$} \\
\hline f4-04 & Powell's Quartic & 4 & $-10 \leq x_{i} \leq 10$ & 0 & {$[26,50]$} \\
\hline$f 4-05$ & NeumaierF2 & 4 & $0 \leq x_{i} \leq n$ & 0 & {$[26,34,55]$} \\
\hline$f 4-06$ & Wood (or Colville) & 4 & $-10 \leq x_{i} \leq 10$ & 0 & {$[30,31,37,50,58]$} \\
\hline f5-01 & AMGM & 5 & $0 \leq x_{i} \leq 10$ & 0 & [34] \\
\hline$f 5-02$ & Osborne F1 & 5 & $\begin{array}{c}0 \leq x_{1}, x_{2}, x_{4}, x_{5} \leq 3 \\
-3 \leq x_{3} \leq 0\end{array}$ & $5.46 \times 10^{-5}$ & {$[63,64]$} \\
\hline$f 5-03$ & SODP & 5 & $-1 \leq x_{i} \leq 1$ & 0 & {$[34,36,37]$} \\
\hline$f 5-04$ & Styblinski-Tang & 5 & $-5 \leq x_{i} \leq 5$ & $-39.16616570377142 n$ & {$[33,34]$} \\
\hline$f 6-01$ & Hartman's F2 & 6 & $0 \leq x_{i} \leq 1$ & -3.32236801141551 & $\begin{array}{l}{[23,26,30,31,32,} \\
34,37,40]\end{array}$ \\
\hline f6-02 & Perm F2 & 6 & $-1 \leq x_{i} \leq 1$ & 0 & [55] \\
\hline f9-01 & ANNs XOR & 9 & $-1 \leq x_{i} \leq 1$ & 0.959759 & [55] \\
\hline f9-02 & Price's Transistor & 9 & $-10 \leq x_{i} \leq 10$ & 0 & [26] \\
\hline f9-03 & Storn'sChebyshev & 9 & $-2^{n} \leq x_{i} \leq 2^{n}$ & 0 & {$[26,59]$} \\
\hline$f 10-01$ & EpistaticMichalewicz & 10 & $0 \leq x_{i} \leq \pi$ & -9.660152 & {$[26,59]$} \\
\hline$f 10-02$ & Katsuura & 10 & $-1000 \leq x_{i} \leq 1000$ & $1($ for $n=10)$ & {$[57]$} \\
\hline$f 10-03$ & Odd Square & 10 & $-15 \leq x_{i} \leq 15$ & $-1.143833($ for $n=10)$ & {$[26,31]$} \\
\hline f10-04 & Paviani & 10 & $-2.001 \leq x_{i} \leq 9.999$ & -45.77848 & {$[26,31,54,60]$} \\
\hline$f 15-01$ & Dixon-Price & 15 & $-10 \leq x_{i} \leq 10$ & 0 & {$[28,34]$} \\
\hline$f 15-02$ & NeumaierF3 (or Trid) & 15 & $-n^{2} \leq x_{i} \leq n^{2}$ & $-665($ for $n=15)$ & {$[26,31,34,48]$} \\
\hline$f 15-03$ & $\begin{array}{c}\text { Normalized Rana's } \\
\text { Function +Diagonal Wrap }\end{array}$ & 15 & $-520 \leq x_{i} \leq 520$ & $\begin{array}{l}-512.753162426239100568636 \\
786193\end{array}$ & {$[60,65]$} \\
\hline$f 17-01$ & Bent Cigar & 17 & $-100 \leq x_{i} \leq 100$ & 0 & [34] \\
\hline f17-02 & $\begin{array}{c}\text { Deflected Corrugated } \\
\text { Spring }\end{array}$ & 17 & $0 \leq x_{i} \leq 10$ & -1 & {$[22,58]$} \\
\hline$f 17-03$ & Infinity (or Csendes) & 17 & $-1 \leq x_{i} \leq 1$ & 0 & [34] \\
\hline f20-01 & Alpine & 20 & $-10 \leq x_{i} \leq 10$ & 0 & {$[34,37]$} \\
\hline f20-02 & Quintic & 20 & $-10 \leq x_{i} \leq 10$ & 0 & {$[34,55]$} \\
\hline f20-03 & Pathological & 20 & $-100 \leq x_{i} \leq 100$ & 0 & [37] \\
\hline$f 30-01$ & Ackley & 30 & $-32 \leq x_{i} \leq 32$ & 0 & $\begin{array}{l}23,26,28,30,31, \\
34,36,37,46,57]\end{array}$ \\
\hline$f 30-02$ & GeneralizedGriewank & 30 & $-600 \leq x_{i} \leq 600$ & 0 & {$[23,26,28,30]$} \\
\hline f30-03 & Generalized Penalized F1 & 30 & $-50 \leq x_{i} \leq 50$ & 0 & {$[23,62]$} \\
\hline f30-04 & Generalized Penalized F2 & 30 & $-50 \leq x_{i} \leq 50$ & 0 & {$[23,62]$} \\
\hline$f 30-05$ & GeneralizedRastrigin & 30 & $-5.12 \leq x_{i} \leq 5.12$ & 0 & $\begin{array}{l}{[22,23,26,28,30,} \\
31,36,37,46,53,57, \\
62]\end{array}$ \\
\hline f30-06 & GeneralizedRosenbrock & 30 & $-30 \leq x_{i} \leq 30$ & 0 & $\begin{array}{l}{[23,26,28,31,32,} \\
36,37,46,62]\end{array}$ \\
\hline
\end{tabular}




\begin{tabular}{|c|c|c|c|c|c|}
\hline$f 30-07$ & $\begin{array}{c}\text { GeneralizedSchwefel } \\
\text { F2.26 }\end{array}$ & 30 & $-500 \leq x_{i} \leq 500$ & $\begin{array}{l}-418.982887272433799807913 \\
601398 n\end{array}$ & $\begin{array}{l}{[22,23,26,28,30,} \\
31,34,36,53,60,62, \\
65]\end{array}$ \\
\hline f30-08 & Mishra F1 & 30 & $0 \leq x_{i} \leq 1$ & 2 & [34] \\
\hline$f 30-09$ & Mishra F2 & 30 & $0 \leq x_{i} \leq 1$ & 2 & [34] \\
\hline$f 30-10$ & $\begin{array}{c}\text { Quartic (or De Jong's } \\
\text { F4) }\end{array}$ & 30 & $-1.28 \leq x_{i} \leq 1.28$ & 0 & {$[23,31,37,46,56]$} \\
\hline$f 30-11$ & Schwefel F1.2 & 30 & $-100 \leq x_{i} \leq 100$ & 0 & {$[23,31,37,53,62]$} \\
\hline$f 30-12$ & Schwefel F2.21 & 30 & $-100 \leq x_{i} \leq 100$ & 0 & {$[23,31,37,62]$} \\
\hline f30-13 & Schwefel F2.22 & 30 & $-10 \leq x_{i} \leq 10$ & 0 & $\begin{array}{l}{[23,30,31,37,53,} \\
62]\end{array}$ \\
\hline f30-14 & $\begin{array}{l}\text { Sphere(Square Sum, } \\
\text { Harmonic or De Jong's } \\
\text { F1) }\end{array}$ & 30 & $-100 \leq x_{i} \leq 100$ & 0 & $\begin{array}{l}{[23,28,31,36,37,} \\
46,53,57,62]\end{array}$ \\
\hline f30-15 & Step & 30 & $-100 \leq x_{i} \leq 100$ & 0 & $\begin{array}{l}{[23,28,31,37,53,} \\
57]\end{array}$ \\
\hline$f 60-01$ & Hyper-Ellipsoid & 60 & $-1 \leq x_{i} \leq 1$ & 0 & [57] \\
\hline $660-02$ & Qing & 60 & $-500 \leq x_{i} \leq 500$ & 0 & {$[53]$} \\
\hline $660-03$ & Salomon & 60 & $-100 \leq x_{i} \leq 100$ & 0 & {$[26,53]$} \\
\hline
\end{tabular}

\section{REFERENCES}

[1] Dan. Simon, "Biogeography-based optimization," IEEE Trans. On Evolutionary Computation, vol. 12, no. 6, pp. 702-713, Dec. 2008.

[2] Robert H. MacArthur and Edward O. Wilson, "An Equilibrium Theory of Insular Zoogeography," Journal of Evolution, vol. 17, no. 4, pp. 373-387, Dec. 1963.

[3] Robert H. MacArthur and Edward O. Wilson, The Theory of Island Biogeography. Princeton, New Jersey: Princeton University Press, 1967.

[4] Mark V. Lomolino, Brett R. Riddle and James H. Brown, Biogeography, 3rd ed. Sunderland, Massachusetts: Sinauer Associates Inc., 2009.

[5] R. J. Lincoln, G. A. Boxshall and P. F. Clark, A dictionary of Ecology, Evolution and Systematics. Cambridge, UK: Cambride University Press, 1982.

[6] PaulamiMaiti and Prabodh K. Maiti, Biodiversity: Perception, Peril and Preservation. Prentice-Hall of India, 2011.

[7] William A. Nierenberg, Encyclopedia of Environmental Biology, vol. 2. San Diego, California: Academic Press Inc., 1995.

[8] Jonathan B. Losos and Robert E. Ricklefs, The Theory of Island Biogeography Revisited. Princeton, New Jersey: Princeton University Press, 2010.

[9] Alan A. Myers and Paul S. Giller, Analytical Biogeography: An Integrated Approach to the Study of Animal and Plant Distributions. London, UK: Chapman and Hall, 1990.

[10] Martin L. Cody, Plants on Islands: Diversity and Dynamics on a Continental Archipelago. Berkeley and Los Angeles, California: University of California Press, 2006.

[11] Robert H. MacArthur and Joseph H. Connell, The Biogeography of Populations. New York: John Wiley \& Sons Inc., 1966.

[12] Robert H. MacArthur, Geographical Ecology: Patterns in the Distribution of Species. New York: Harper \& Row Publishers Inc., 1972.

[13] Haiping Ma, "An analysis of the equilibrium of migration models for biogeography-based optimization," Information Sciences, vol. 180, no. 18, pp. 3444-3464, 15 Sept. 2010.

[14] Haiping Ma and Dan. Simon, "Blended biogeography-based optimization for constrained optimization," Engineering Applications of Artificial Intelligence, vol. 24, no. 3, pp. 517-525, Apr. 2011.

[15] Dan. Simon, "A probabilistic analysis of a simplified biogeography-based optimization algorithm," Evolutionary Computation, vol. 19, no. 2, pp. 167-188, Summer 2011.

[16] Haiping Ma and Dan. Simon, "Analysis of migration models of biogeography-based optimization using Markov theory," Engineering Applications of Artificial Intelligence, vol. 24, no. 6, pp. 1052-1060, Sept. 2011.

[17] Dan. Simon, M. Ergezer and Dawei Du, "Population distributions in biogeography-based optimization algorithms with elitism," IEEE International Conference on Systems, Man and Cybernetics, pp. 991-996, Oct. 2009. 
[18] Wenying Gong, ZhihuaCai and Charles X. Ling, "DE/BBO: A hybrid differential evolution with biogeography-based optimization for global numerical optimization," Soft Computing, vol. 15, no. 4, pp. 645-665, Apr. 2011.

[19] James A. McMahon, "Disturbed lands and ecological theory: an essay about a mutualistic association," in: Restoration Ecology, a Synthetic Approach to Ecological Research, William R. Jordan III, Michael E. Gilpin and John D. Aber. Cambridge, UK: Cambride University Press, 1987, pp. 221-237.

[20] Ali R. Alroomi, Fadhel A. Albasri and Jawad H. Talaq, "Performance Comparison between the Original Forms of Biogeography-Based Optimization," to be published, Sept. 2013.

[21] Dan. Simon, "The Matlab Code of Biogeography-Based Optimization," [online] Aug. 2008, http://academic.csuohio.edu/simond/bbo/(Accessed: 01 Feb. 2013).

[22] Wen Wan and Jeffrey B. Birch, "Using a modified genetic algorithm to find feasible regions of a desirability function," Quality and Reliability Engineering International, vol. 27, no. 8, pp. 1173-1182, Dec. 2011.

[23] Xin Yao, Yong Liu and Guangming Lin, "Evolutionary Programming Made faster," IEEE Trans. on Evolutionary Computation, vol. 3, no. 2, pp. 82-102, Jul. 1999.

[24] Chien-Wen Chao, Shu-CherngFand and Ching-Jong Liao, "A Tropical cyclone-based method for global optimization," Journal of Industrial and Management Optimization, Vol. 8, no. 1, Feb. 2012.

[25] Elena Kiseleva and Tatyana Stepanchuk, "On the Efficiency of a Global Non-differentiable Optimization Algorithm Based on the Method of Optimal Set Partitioning," Journal of Global Optimization, vol. 25, no. 2, pp. 209-235, Feb. 2003.

[26] M. Montaz Ali, CharoenchaiKhompatraporn and Zelda B. Zabinsky, "A Numerical Evaluation of Several Stochastic Algorithms on Selected Continuous Global Optimization Test Problems" Journal of Global Optimization, vol. 31, no. 4, pp. 635-672, Apr. 2005.

[27] Ray-Bing Chen, Weichung Wang and Fuhuang Tsai, "A Basis-based Response Surface Method for Computer Experiment Optimization," 2006

[28] Milos Subotic, Milan Tuba and NadezdaStanarevic, "Different approaches in parallelization of the artificial bee colony algorithm," International Journal of Mathematical and Methods in Applied Sciences, vol. 5, no. 4, Mar. 2011.

[29] Celso A. G. Santos, Paula K. M. M. Freire and Sudhanshu K. Mishra, "Cuckoo search via Le' vy Flights for optimization of a physically-based runoff-erosion model," Journal of Urban and Environmental Engineering, vol. 6, no. 2 , pp. 123-131, Dec. 2012

[30] Xinchao Zhao and Xiao-Shan Gao, "Affinity genetic algorithm," Journal of Heuristics, vol. 13, no. 2, pp. 133-150, Apr. 2007.

[31] Ernesto P. Adorio. (2005, Jan.), MVF - Multivariate Test Functions Library in C for Unconstrained Global Optimization. University of the Philippines Diliman. Quezon City, Metro Manila, Philippines. Available: http://geocities.ws/eadorio/mvf.pdf

[32] RachidChelouah and Patrick Siarry, "Tabu Search applied to global optimization," European Journal of Operational Research, vol. 123, no. 2, pp. 256-270, Jun. 2000.

[33] Sudhanshu Mishra. (2006, Aug.). Some new test functions for global optimization and performance of repulsive particle swarm method. North-Eastern Hill University. Shillong, India. Available: http://mpra.ub.uni-muenchen.de/2718/1/MPRA_paper_2718.pdf

[34] Andrea Gavana, "Test Functions Index," [online] Feb. 2013, http://infinity77.net/ global_optimization/test_functions.html (Accessed: 01 April 2013).

[35] Z. Emami, M. H. Farahi and E. Ansari. (2012, Feb.). Global Optimization with Electro Weak. The Open Industrial and Manufacturing Engineering Journal. [Online]. vol. 5, pp. 1-7. Available: http://benthamscience.com/open/toimej/articles/V005/1TOIMEJ.pdf

[36] MarcinMolga and CzesławSmutnicki. (2005, Apr.). Test functions for optimization needs. North-Eastern Hill University. Shillong, India. Available: http://www.zsd.ict.pwr.wroc.pl/files/ docs/functions.pdf

[37] ShahryarRahnamayan, Hamid R. Tizhoosh and Magdy M. A. Salama, "A novel population initialization method for accelerating evolutionary algorithms," Computers \& Mathematics with Applications, vol. 53, no. 10 , pp. 1605-1614, May 2007

[38] Leo Breiman and Adele Cutler, "A deterministic algorithm for global optimization," Mathematical Programming, vol. 58, no.1-3, pp. 179-199, Jan. 1993.

[39] Mohamed B. Trabia and Xiao Bin Lu, "A Fuzzy Adaptive Simplex Search Optimization Algorithm," Journal of Mechanical Design, vol. 123, no. 2, pp. 1-10, Jun. 2001.

[40] J. B. Lee and B. C. Lee, "A global optimization algorithm based on the new filled function method and the genetic algorithm," Engineering Optimization, vol. 27, no. 1, pp. 1-20, 1996.

[41] M. Fernanda P. Costa, Florbela P. Fernandes, Edite Manuela da G. P. Fernandes, "A deterministicstochastic method for nonconvex MINLP problems," 2nd International Conference on Engineering Optimization, Sept. 6-9, 2010, Lisbon, Portugal. 
[42] Kaj Madsen, "Test problems for global optimization," [online] Jun. 2008, http://www2.imm. dtu.dk/ kajm/Test_ex_forms/test_ex.html (Accessed: 09 April 2013).

[43] M. N. Vrahatis, D. G. Sotiropoulos and E. C. Triantafyllou, "Global optimization for imprecise problems," in: Developments in Global Optimization, Nonconvex Optimization and Its Applications, Immanuel M. Bomze, TiborCsendes, Reiner Horst, Panos M. Pardalos. Springer Science+Business Media Dordrecht: Kluwer Acdemic Publishers, 1997, vol. 18, pp. 37-54.

[44] Baker Kearfott, "An efficient degree-computation method for a generalized method of bisection," NumerischeMathematik, vol. 32, no. 2, pp. 109-127, 1979.

[45] EsinOnbaşoğlu and LinetÖzdamar, "Parallel Simulated Annealing Algorithms in Global Optimization," Journal of Global Optimization, vol. 19, no. 1, pp. 27-50, Jan. 2001.

[46] S. Rahnamayan, H. R. Tizhoosh and M. M. A. Salama, "Opposition-Based Differential Evolution for Optimization of Noisy Problems," IEEE Congress on Evolutionary Computation, CEC 2006, pp.18651872, Jul. 2006.

[47] Klaus Müller and Leo D. Brown, "Location of saddle points and minimum energy paths by a constrained simplex optimization procedure," Theoreticachimicaacta, vol. 53, no. 1, pp. 75-93, 1979.

[48] Wan-Ching Chou, "Multiple Optima Problems in Higher Dimensions," [online] Mar. 2013,http://www.math.ntu.edu.tw/ wwang/cola_lab/test_problems/multiple_opt/multiopt_prob/ (Accessed: 25 April 2013).

[49] MathWorks, "Global Optimization Toolbox," [online] 2013, http://www.mathworks.com/ products/globaloptimization/index.html (Accessed: 25 April 2013).

[50] Singiresu S. Rao, Engineering Optimization, Theory and Practice, 4th ed. Hoboken, New Jersey: John Wiley \& Sons Inc., 2009.

[51] Gisela C.V. Ramadas and Edite M.G.P. Fernandes, "Solving Nonlinear Equations by a Tabu Search Strategy," 11th International Conference on Computational and Mathematical Methods in Science and Engineering, CMMSE2011, vol. 4, no. 1, pp. 1578-1589, Jun. 2011.

[52] Jorge J. Moré, Burton S. Garbow and Kenneth E. Hillstrom, "Testing Unconstrained Optimization," ACM Transactions on Mathematical Software (TOMS), vol. 7, no. 1, pp. 17-41, Mar. 1981.

[53] Anyong Qing, Differential Evolution, fundamentals and Applications in Electrical Engineering. Singapore: Wiley-IEEE Press, Sept. 2009.

[54] Muhammad Aria, "Educational Simulator for Teaching of Particle Swarm Optimization in LabVIEW," TELEKONTRAN, vol. 1, no. 1, Jan. 2013.

[55] Sudhanshu Mishra. (2006, Oct.). Repulsive Particle Swarm Method on Some Difficult Test Problems of Global Optimization. North-Eastern Hill University. Shillong, India. Available: http://mpra.ub.unimuenchen.de/1742/1/MPRA_paper_1742.pdf

[56] V. Zitko. (1984, Sept.), "Simplex Optimization," Canadian Technical Report of Fisheries and Aquatic Sciences, No. 1308. Fisheries and Oceans. Biological Station (St. Andrews, N.B.), Canada. Available: http://www.dfo-mpo.gc.ca/Library/20701.pdf

[57] Rainer Storn and Kenneth Price, "Differential Evolution - A Simple and Efficient Heuristic for Global Optimization over Continuous Spaces," Journal of Global Optimization, vol. 11, no. 4, pp. 341-359, Dec. 1997.

[58] Ashok D. Belegundu and Tirupathi R. Chandrupatla, Optimization Concepts and Applications in Engineering, 2nd ed. New York: Cambridge University Press, 2011. Page331

[59] Kenneth V. Price, Rainer M. Storn and Jouni A. Lampinen, Differential Evolution: A Practical Approach to Global Optimization, Berlin, Germany: Springer Science+Business Media, 2005.

[60] Sudhanshu Mishra. (2012, Aug.). Global optimization of some difficult benchmark functions by cuckoohost co-evolution meta-heuristics. North-Eastern Hill University. Shillong, India. Available: http://mpra.ub.uni-muenchen.de/40666/1/MPRA_paper_40666.pdf

[61] Darrell Whitley, Deon Garrett and Jean-Paul Watson, "Quad Search and Hybrid Genetic Algorithms," in: Genetic and Evolutionary Computation--GECCO 2003, G. Goos, J. Hartmanis and J. Van Leeuwen. Berlin, Germany: Springer Science+Business Media, 2003, vol. 2, pp. 1469-1480.

[62] A. K. Qin, V. L. Huang and P. N. Suganthan, "Differential Evolution Algorithm with Strategy Adaptation for Global Numerical Optimization," IEEE Trans. On Evolutionary Computation, vol. 13, no. 2, pp. 398 417, Apr. 2009.

[63] David R. Monismith Jr., "The Uses of the Slime Mold Lifecycle as a Model for Numerical Optimization," Ph.D. dissertation, Dept. Elect. Eng., Oklahoma State University, Oklahoma City, OK, 2010.

[64] DebasisKundu and Amit Mitra, "Estimating the parameters of the linear compartment model," Journal of Statistical Planning and Inference, vol. 70, no. 2, pp. 317-334, May 1998.

[65] Lappeenranta University of Technology, "The function testbed," [online] May. 2007, http://www.it.lut.fi/ip/evo/functions/functions.html (Accessed: 10 April 2013). 


\section{Authors}

Ali Ridha Alroomi received the B.Sc. degree in Process Instrumentation and Control Engineering from University of Bahrain, Bahrain, in 2006. In the period between 20092013, he covered the electrical courses of the B.Sc. program in Electrical Engineering, and followed by M.Sc. degree from the same university. After B.Sc. graduation, he was selected to be as a project engineer in Moore Control \& Engineering "MCE Middle East" till the mid of 2007, when he joined Yokogawa Middle East as a DCS subsystem and graphics engineer. From 2008 to 2012, he worked in Aluminum Bahrain (ALBA B.SC.)

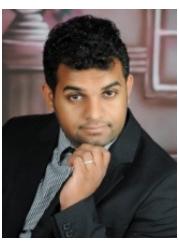
as an instrumentation and control engineer in its power plants, generation and auxiliary C\&I maintenance. $\mathrm{He}$ is currently working as a researcher in the field of electrical power systems and evolutionary computation.

Fadhel Abbas Al-Basri received the B.Sc. and M.Sc. degrees in Electrical Engineeringfrom University ofBahrain, Bahrain, and Ph.D. degree in the same field from University of Western Ontario, Canada,in 1992, 1997 and 2007, respectively. He worked in Ministry of Electricity and Water, Bahrain, as an electrical engineer from 1993 to 1994. In 1994, he joined the University ofBahrain as teaching and research assistant and currently an assistant professor in the department of electrical engineering. His research interest is power systems protection, power systems analysis andFACTS-devices.

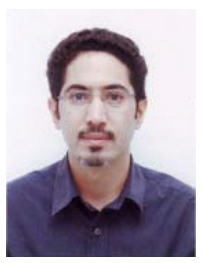

Jawad Hasan Talaq received B.Sc. degree from University of Technology, Baghdad, Iraq (1981), M.Sc. degree from University of Strathclyde,Glasgow, UK (1987), and Ph.D. degree from Technical University of Nova Scotia, Halifax, Canada (1993); all in electrical engineering. He worked in Ministry of Electricity and Water, Bahrain, as a graduate engineer in Riffa power plant from 1981 to 1985, and as a shift charge engineer from 1985 to 1989 , when he moved from industrial field to academic field. He is now an associate professor in the department of electrical engineering, University of Bahrain. His

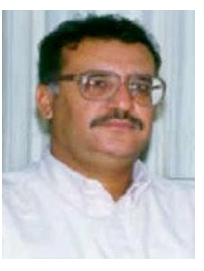
teaching covers power systems operation and control, power systems dynamics, and advanced power systems analysis; and his research topics include power systems operation, control and dynamics, and applied soft computing. 